\title{
Enhancing finite differences with radial basis functions: Experiments on the Navier-Stokes equations
}

\author{
Natasha Flyer \\ National Center for Atmospheric Research \\ Institute for Mathematics Applied to Geosciences \\ Boulder, CO 80305 USA \\ flyer@ucar.edu* \\ Gregory A. Barnett \\ University of Colorado \\ Department of Applied Mathematics \\ Boulder, CO 80309 USA \\ gregory.barnett@colorado.edu \\ Louis J. Wicker \\ NOAA National Severe Storms Laboratory \\ Norman, OK 73072 USA \\ louis.wicker@noaa.gov
}

February 21, 2016

\begin{abstract}
Polynomials are used together with polyharmonic spline (PHS) radial basis functions (RBFs) to create local RBF-finite-difference (RBF-FD) weights on different node layouts for spatial discretizations that can be viewed as enhancements of the classical finite differences (FD). The presented method replicates the convergence properties of FD but for arbitrary node layouts. It is tested on the 2D compressible Navier-Stokes equations at low Mach number, relevant to atmospheric flows. Test cases are taken from the numerical weather prediction community and solved on bounded domains. Thus, attention is given on how to handle boundaries with the RBF-FD method, as well as a novel implementation for hyperviscosity. Comparisons are done on Cartesian, hexagonal, and quasi-uniform node layouts. Consideration and guidelines are given on PHS order, polynomial degree and stencil size. The main advantages of the present method are: 1) capturing the basic physics of the problem surprisingly well, even at very coarse resolutions, 2) high-order accuracy without the need of tuning a shape parameter, and 3) the inclusion of polynomials eliminates stagnation (saturation) errors. A MATLAB code is given to calculate the differentiation weights for this novel approach.
\end{abstract}

\section{Background and Introduction}

A new numerical approach, within the RBF-FD framework, is introduced with the goal being:

${ }^{*}$ Corresponding author

(C) 2016. This manuscript version is made available under the Elsevier user license http://www.elsevier.com/open-access/userlicense/1.0/ 
To enhance classical finite differences (FD) to arbitrary node layouts in multidimensions by augmenting polyharmonic spline (PHS) RBFs. The consequences are that the convergence rate is completely governed by the degree of polynomials used as in FD, while the augmented PHS RBFs create a modified basis that is non-singular on any node layout in any number of dimensions.

From an approximation theory standpoint, it is important to note the following two points. The first is with regard to why we use PHS RBFs, the second is with regard to why we need to augment RBFs to polynomials for arbitrary node layouts. Together they form the underlying foundation of this novel approach:

1. Augmenting the polynomial basis with infinitely smooth RBFs such as GA, will have little influence on the convergence rate. The reason being is that as infinitely smooth RBFs go flat they span the same space as polynomials. A good illustration of this is Figures 2 and 3 in [19]. PHS, $r^{2 m-1}$ or $r^{2 m} \log r$ for $m \in \mathbb{N}_{+}$where $r$ is the Euclidean distance between where the $\mathrm{RBF}$ is centered and where is evaluated, have jumps in derivatives and do not span the space of polynomials.

2. The Mairhuber-Curtis Theorem of 1956 [26, 46, 10, 17] tells that no set of data-independent basis functions (such as polynomials, Fourier, Legendre, spherical harmonics, etc.) can provide non-singular interpolation for distinct node locations beyond 1-D. In the framework of polynomials, this implies (i) that standard FD formulas must be confined to lattice-based grids, generally using tensor products and ii) to achieve a well-posed multivariate data interpolation problem (and hence calculate derivative operators) on non-lattice-based points (i.e. any arbitrary node layout), the basis needs to depend on the data locations. Augmenting PHS RBFs allows for this.

From a historical perspective, before the development of RBF-FD or other flavors of local RBFs $[38,39,42]$, applications of RBFs were global, not considering the current approach most likely for the following reasons:

1. If piecewise smooth RBFs were used, they were used in conjunction with low-order polynomials, e.g $1, x, y$ in 2-D. The only role of the polynomial was to guarantee non-singularity of the RBF interpolation matrix for very unusual node layouts, which needs to be inverted to derive the differentiation matrices $[46,10]$. The role of capturing the physics of complicated fluid flows was the left to the RBFs.

2. Furthermore, non-smooth PHS RBFs were not as popular as infinitely smooth RBFs. For example, $r^{3}$ jumps in the third derivative, giving at best fourth-order accuracy in 1-D (with the order of convergence increasing as the dimension increases (c.f. [33]) assuming smooth data). The curse lies in the fact that as $m$ increases, leading to a smoother RBF, the condition number of the interpolation matrix gravely increases. Thus, in the past, one was limited to keeping $m$ small and having low algebraic accuracy.

3. Lastly, using polynomials on a global scale can be dangerous, since it can lead to Runge phenomena near the boundaries. In contrast, on a local scale as in the RBF-FD method, one is only interested in the approximation at the center of the stencil and not at the edges.

As a result, a new way to use PHS RBFs combined with polynomials in the context of RBFFD is introduced, such that high-order accuracy is gained with excellent conditioning of RBF-FD interpolation matrix and no saturation error is encountered. Furthermore, there is no need to 
bother with selecting an optimal shape parameter, which plays an important role in the accuracy of the solution when using infinitely smooth RBFs $[11,2,6,37,5])$. We will demonstrate the performance of the modified RBF-FD method for 1) the 2D advection of a scalar in a strong shear flow (a hyperbolic PDE introduced by [29] and popularized by [3]) and 2) the 2D nonhydrostatic compressible Navier-Stokes on bounded domains applied to test cases common in the numerical weather prediction community. Although already broad in scope, the authors further wish to classify the differences, if any, that occur in applying this methodology on different node layouts: 1) Cartesian, 2) hexagonal, and 3) quasi-uniform. The rationale being that classical finite difference methods, based on polynomials, are usually implemented on Cartesian lattices; hexagonal layouts are optimal in terms of node packing in 2D, supplying information along 3 distinct directions in contrast to Cartesian layouts where information is aligned only along 2 directions; and quasi-uniform nodes allow for geometric flexibility of the domain and the ability of node refinement.

The paper is organized as follows: Section 2 very briefly introduces RBFs. Section 3 discusses the calculation of RBF-FD weights using polynomials. Section 4 demonstrates how the inclusion of polynomials with PHS eliminates stagnation (saturation) errors. Section 5 discusses the node sets that are used and how boundaries and hyperviscosity are handled. Section 6 applies the methodology on the various node layouts, giving detailed results from test cases that are standard in the numerical weather prediction community. Lastly, Section 7 summarizes the observations of this paper.

\section{A brief introduction to Radial Basis Functions}

An $\mathrm{RBF}$ is a $d$-dimensional radially symmetric function $\phi: \mathbb{R}^{d} \rightarrow \mathbb{R}$ that depends only on the Euclidean distance between where the RBF is centered, $\mathbf{x}_{k}$ and where it is evaluated, $\mathbf{x}$. That is, regardless of dimension, its argument is always a scalar defined by $r=\left\|\mathbf{x}-\mathbf{x}_{k}\right\|_{2}$, where $\|\cdot\|_{2}$ denotes the Euclidean distance. RBFs come in two flavors: 1) piecewise smooth and 2) infinitely smooth. The former features a jump in some derivative and thus can only lead to algebraic convergence. The latter is a $C^{\infty}$ function and can lead to spectral convergence when the data is sufficiently smooth. Only PHS RBFs do not depend on a parameter $\varepsilon$ that controls the shape of the RBF (which influences both the conditioning of the matrices and the accuracy of the results $[44,35]$ ). This last comment is a strong motivation of this paper since, by using PHS RBF, one avoids the difficulty of dealing with a shape parameter and yet can achieve high-order accuracy. For theoretical aspects of PHS (a class of conditionally positive definite radial functions), see [10, 46]. Common RBFs of both categories are given in Table 1 , where $\mathbf{x}_{k}$ represents where the RBF is centered.

\section{Calculating PHS-polynomial RBF-FD differentiation weights}

Let us assume we want to approximate a linear operator $L$, e.g. $L=\frac{\partial}{\partial y}$, at a node point $x_{c}$ in the domain by a linear combination of the function values, $\left\{u_{k}\right\}_{k=1}^{n}$, at the nearest $n$ node locations, $\left\{\mathbf{x}_{k}\right\}_{k=1}^{n}$ (with the node $\mathbf{x}_{c}$ itself included in the count; this group of nodes compose the stencil). In other words, we seek weights such that

$$
\sum_{k=1}^{n} w_{k} u_{k}=\left.(L u)\right|_{x=x_{c}}
$$

with $n$ and $w_{k}$ being known as the stencil size and differentiation weights, respectively. To calculate $w_{k}$, we enforce that this linear combination of function values be exact for the interpolant $s(\mathbf{x})$ of 
Table 1: Some common choices for radial functions $\phi(r)$

\begin{tabular}{ll}
\hline Piecewise smooth RBFs & $\phi\left(r=\left\|\mathbf{x}-\mathbf{x}_{k}\right\|_{2}\right)$ \\
\hline Polyharmonic Splines (PHS) [8] & $r^{2 m} \log r, m \in \mathbb{N}$ \\
& $r^{2 m+1}, m \in \mathbb{N}^{0}$ \\
Matern [30] & $\frac{2^{1-m}}{\Gamma(m)} r^{m} K_{m}(\varepsilon r), m>0, \quad$ (Bessel $K$-function) \\
Compact support ('Wendland' [45]) & $(1-\varepsilon r)_{+}^{m} p(\varepsilon r), p$ certain polynomials, $m \in N$ \\
\hline Infinitely smooth RBFs & \\
\hline Gaussian (GA) & $e^{-(\varepsilon r)^{2}}$ \\
Multiquadric (MQ) & $\sqrt{1+(\varepsilon r)^{2}}$ \\
Inverse Multiquadric (IMQ) & $1 / \sqrt{1+(\varepsilon r)^{2}}$ \\
Inverse Quadratic (IQ) & $1 /\left(1+(\varepsilon r)^{2}\right)$ \\
\hline
\end{tabular}

the form

$$
s(\mathbf{x})=\sum_{k=1}^{n} \lambda_{k} \phi\left(\left\|\mathbf{x}-\mathbf{x}_{k}\right\|_{2}\right)+\sum_{k=1}^{l} \mu_{k} p_{k}(\mathbf{x})
$$

with the constraints

$$
\sum_{k=1}^{n} \lambda_{k} p_{j}\left(\mathbf{x}_{\mathbf{k}}\right)=0 \quad j=0,1,2, \ldots, l,
$$

where $p_{l}(\mathbf{x})$ are all multivariate polynomials up to degree $l$ in the dimension of the problem and $\phi\left(\left\|\mathbf{x}-\mathbf{x}_{k}\right\|_{2}\right)$ is a PHS centered at the node $x_{k}$. Classical FD would enforce that it be exact for a polynomial interpolant using a tensor product of univariate bases instead. The constraints enforce that the RBF basis reproduces polynomials up to degree $l$, as well as ensure that the far-field RBF expansion is regularized (i.e. does not blow up) [15]. They also are known as the vanishing moment conditions [25]. From a theoretical aspect, the addition of polynomials also guarantees that under very uncommon node layouts the interpolation problem has a unique solution, given that the node set is unisolvent with respect to the polynomials.

As the simplest example of calculating differentiation weights, let us consider approximating $L=\frac{\partial}{\partial y}$ with PHS $r^{3}$ and polynomials up to degree $l=1$ in 2 D. This leads to the following linear system (note that much higher-order polynomials are used in the numerical method)

$$
\left[\begin{array}{ccc|ccc}
\left\|\mathbf{x}_{1}-\mathbf{x}_{1}\right\|_{2}^{3} & \cdots & \left\|\mathbf{x}_{1}-\mathbf{x}_{n}\right\|_{2}^{3} & 1 & x_{1} & y_{1} \\
\vdots & \ddots & \vdots & \vdots & \vdots & \vdots \\
\left\|\mathbf{x}_{n}-\mathbf{x}_{1}\right\|_{2}^{3} & \cdots & \left\|\mathbf{x}_{n}-\mathbf{x}_{n}\right\|_{2}^{3} & 1 & x_{n} & y_{n} \\
\hline 1 & \cdots & 1 & 0 & 0 & 0 \\
x_{1} & \cdots & x_{n} & 0 & 0 & 0 \\
y_{1} & \cdots & y_{n} & 0 & 0 & 0
\end{array}\right]\left[\begin{array}{c}
w_{1} \\
\vdots \\
w_{n} \\
\hline w_{n+1} \\
w_{n+2} \\
w_{n+3}
\end{array}\right]=\left[\begin{array}{c}
\left.L\left\|\mathbf{x}-\mathbf{x}_{1}\right\|_{2}^{3}\right|_{\mathbf{x}=\mathbf{x}_{c}} \\
\vdots \\
\left.L\left\|\mathbf{x}-\mathbf{x}_{n}\right\|_{2}^{3}\right|_{\mathbf{x}=\mathbf{x}_{c}} \\
\hline\left.L 1\right|_{\mathbf{x}=\mathbf{x}_{c}}=0 \\
\left.L x\right|_{\mathbf{x}=\mathbf{x}_{c}}=0 \\
\left.L y\right|_{\mathbf{x}=\mathbf{x}_{c}}=1
\end{array}\right] .
$$

The weights $w_{n+1}$ to $w_{n+3}$ are ignored after the matrix is inverted. Solving (3) will give one row of the differentiation matrix (DM) that contains the weights for approximating $L$ at $x_{c}$. A MATLAB code to calculate differentiation weights for $\frac{\partial}{\partial x}, \frac{\partial}{\partial y}$, and Laplacian $\Delta$ in $2 \mathrm{D}$ for a single stencil is given in Appendix B. Thus this function would be embedded in a 'for' loop, repeated $N$ times over all nodes in the domain, giving a preprocessing cost of $O\left(n^{3} N\right)$. Since $n<<N$, it should be noted that the DM usually becomes over $99 \%$ zeros. As a result, we do not actually store the DM 
but only its nonzero entries. For further in depth details on RBF-FD weights, see Section 5.1.4 in $[17]$.

\section{Stagnation error, PHS order, and polynomial degree}

Stagnation (saturation) error is defined as the error failing to converge under refinement (this can be seen through the convergence rate either stagnating or decreasing as resolution increases). For infinitely smooth RBFs, as the resolution increases (i.e. $r$ decreases), the shape parameter must increase to maintain the condition number of the matrix in (3), resulting in stagnation error since the more peaked the infinitely smooth RBFs become as $\varepsilon$ increases, the less accurate the approximation. The matrix conditioning problem can be circumvented by calculating in the small $\varepsilon$ regime with a stable algorithm $[19,27]$ but then adding polynomials to infinitely smooth RBFs offer little benefit, since the RBFs themselves virtually span the same function space and exactly the same polynomial function space in the $\varepsilon \rightarrow 0$ limit $[7,20,36]$. In contrast, PHS RBF with polynomials can achieve high-order algebraic convergence without encountering saturation error or the difficulty of finding an optimal value of the shape parameter $\varepsilon$ for good accuracy, which has been a central focus of quite a few studies $[14,24,11,2,6]$. It should be noted that if polynomials are not included with the PHS RBF, stagnation error is encountered since boundary errors at the edge of the RBF-FD stencil can be quite large and penetrate toward the center of the stencil where the interpolant or any derivative is being approximated. Further investigation of the effects of adding polynomials to RBF-FD approximations for both infinitely smooth and PHS RBFs is given in $[12]$.

Since locally all smooth functions are well represented by Taylor expansions, then under refinement, the RBF-FD approximation must reproduce polynomial behavior. In the following numerical studies, it indeed was found that the convergence rate is dictated not by the order of the PHS, $m$, but by the highest degree of polynomials, $l$, used. In addition, for PDEs with only first-order spatial derivatives, as in all the test cases, the convergence rate can be expected to be $O\left(h^{l}\right)$. The reason being is that the error for polynomial interpolation is $O\left(h^{l+1}\right)$, but one order in $h$ is lost in approximating a first derivative. These observations are in excellent agreement with Figure 1, where $d / d x$ of the smooth function $f(x, y)=1+\sin (4 x)+\cos (3 x)+\sin (2 y)$ is approximated with two different PHS RBFs, $r^{3}$ and $r^{7}$, augmented with polynomials (e.g. poly 3 is augmentation of the matrix in (3) with the 10 polynomials, $1, x, y, x^{2}, x y, y^{2}, \ldots$ etc up to degree 3 in $\left.2 \mathrm{D}\right)$. The approximation is at the center of 37 node hexagonal stencil (see Appendix A) with the evaluation nodes of the derivative in its vicinity. The slopes in the two panels of Figure 1 are identical, the only difference being that the constant that multiplies the order of convergence is slightly smaller

for $r^{7}$, thus giving a marginally higher accuracy for a given resolution. It is still important to note that the RBFs play a crucial role in safety against singularities due to particular node layouts.

For the interplay of stencil size and polynomial degree on accuracy, see Section 6.1.2. 

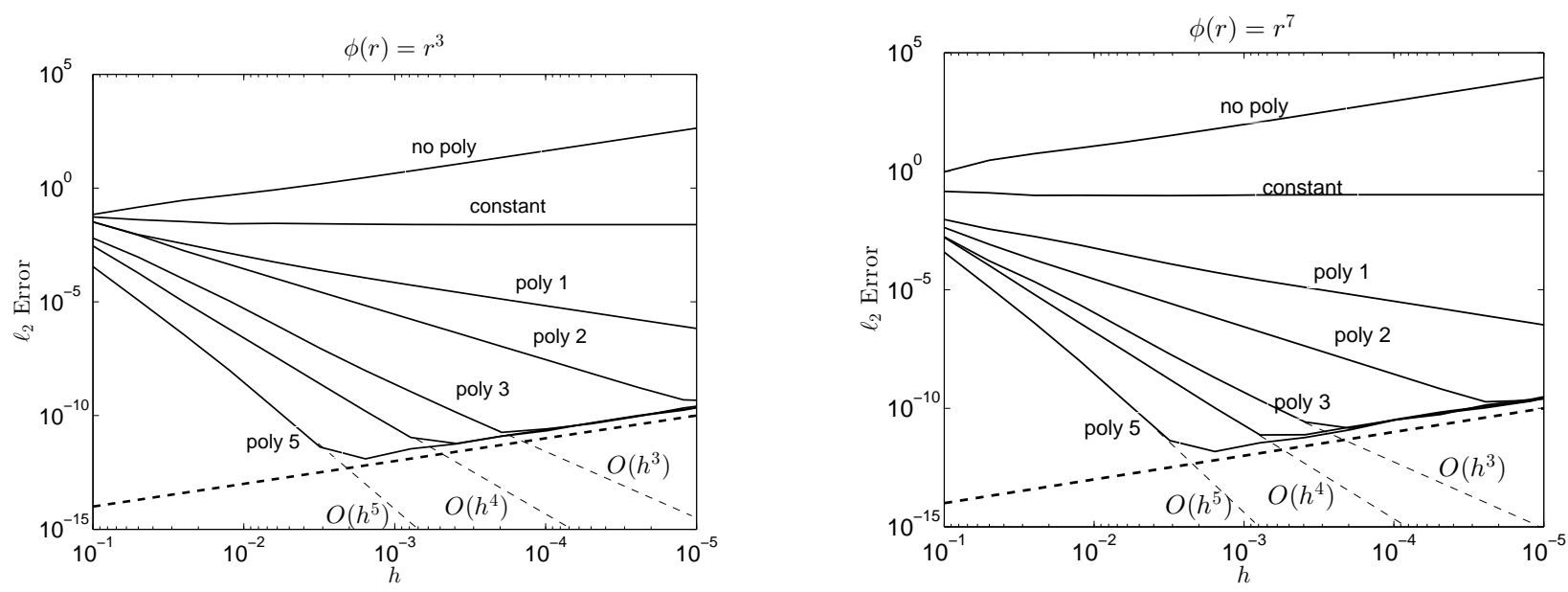

Figure 1: The convergence rate in approximating $d / d x$ of the function $f(x, y)=1+\sin (4 x)+$ $\cos (3 x)+\sin (2 y)$ on a 37 node RBF-FD stencil based on $r^{3}$ and $r^{7}$, augmented with polynomials as described in the text. The dashed line marks machine round-off errors in standard double precision of $10^{-15} / h$ for approximating the first derivative.

\section{Node sets, Ghost nodes, and Hyperviscosity}

Section 5.1 overviews the various node sets considered in this study. Section 5.2 discusses how to increase accuracy near boundaries with the use of ghost nodes and Section 5.3 discusses the need for and type of hyperviscosity used, introducing a novel way of implementing hyperviscosity with PHS and polynomials.

\section{$5.1 \quad$ Node-sets}

Unlike traditional FD methods, the presented local polynomial-RBF method has the advantage of being equally simple to apply on any set of nodes. Figure 2 shows the three types of node-layouts that will be considered in the present study.

1. Cartesian layouts as they are the lattices classical finite differences (FD) are usually implemented on.

2. Hexagonal layouts as they are the optimal packing strategy in 2D (i.e. for a fixed area and inter-nodal distance, this layout provides the densest possible node layout). They have also been considered an optimal layout for differentiation stencils, since where the weights pick up information is aligned along three different directions as opposed to only two with Cartesian layouts. Although FD have been sporadically implemented over the decades on hexagonal node layouts, they have never caught favor due to the complexity of how to handle boundary stencils to best approximate non-symmetric operators, such as $\frac{\partial}{\partial x}$ or $\frac{\partial}{\partial y}$, since both of these cannot be aligned with the primary grid directions leading to anisotropy and to heavy dispersion errors in the solution. As a result, FD applications using hexagonal grids tend to be applied to PDEs with symmetric operators as the Laplacian (e.g. two-way elastic or acoustic wave equations) that can be implemented along the 3 diagonals of a 7 point hex stencil [9, 23, 28]. 
3. Lastly, quasi-uniform node layouts (i.e. nodes do not align along any direction, but on a local zoomed-in level they roughly form hexagonal-type patterns) are considered as they have the great advantage of geometric flexibility that will be needed in future applications on irregular domains and/or with local node refinement. They are generated, starting from a random node distribution $(\operatorname{rand}(\mathrm{N}, 2)$ is the command used in Matlab), via electrostatic repulsion using only the direction vector to move the nodes a preset amount but not the force. However, in current studies for future publications, a front advancing algorithm is used to generate the quasi-uniform as well as variable density node distributions. The code is given in the appendix of [16].
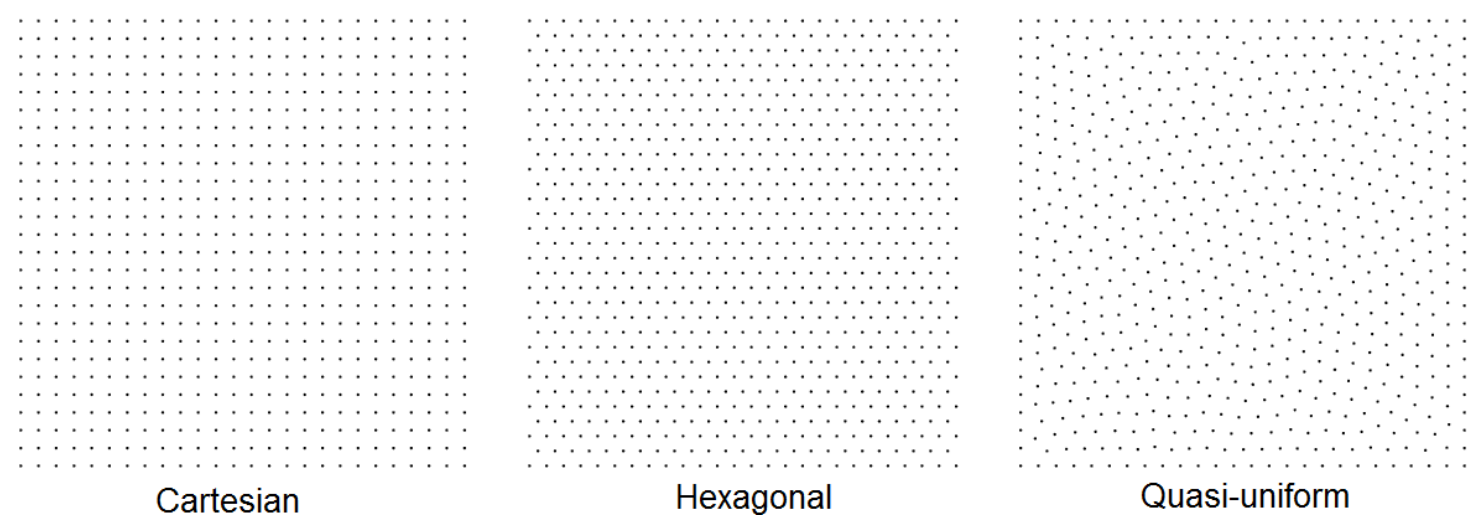

Figure 2: Three different types of node-distributions that are used to solve the $2 \mathrm{D}$ test problems.

\subsection{Ghost nodes}

Near boundaries, stencils become one-sided, leading to a deterioration of the approximation due to Runge phenomenon. In order to ameliorate this effect, one layer of ghost nodes is placed outside the domain at a distance roughly continuing the pattern of the interior nodes. Once the ghost nodes are placed, function values at these locations can be solved for by enforcing additional constraints at the boundary nodes. For example, if the upper boundary of a rectangular domain is free-slip, then $\partial u / \partial z=0$, and this condition can be used to solve for values of $u$ at the ghost nodes. Similarly, the PDE itself can be enforced on the boundary, giving an extra constraint.

\begin{tabular}{|lc|}
\hline Interior Node & 0 \\
Boundary Node & $\bullet$ \\
Ghost Node & 0 \\
Evaluation Point & \\
Stencil Boundary &.--- \\
\hline
\end{tabular}

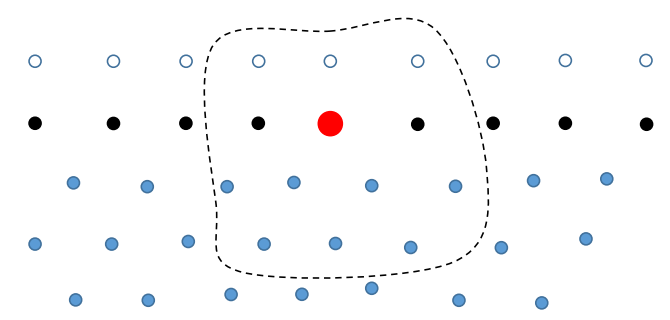

Figure 3: An example of an RBF-FD boundary stencil that might be used for enforcing $\partial u / \partial z=0$ on the top boundary. For boundary stencils, the stencil center can be considered the evaluation point.

For each node on the top boundary, one ghost node is placed just outside the boundary, as shown in Figure 3. The function values for $u$ at the ghost nodes are obtained by enforcing $\partial u / \partial z=0$ 
at each of the top boundary nodes simultaneously. This will lead to a coupled system with as many equations as there are ghost nodes. In the case illustrated in Figure 3, the system will be tridiagonal, as there are three unknown values at the ghost points for each evaluation node on the boundary.

The following is a more detailed discussion on how the ghost node values are calculated. Suppose there are $N_{I}$ interior nodes, $N_{B}$ boundary nodes, and $N_{G}$ ghost nodes, and let the total number of nodes be $N=N_{I}+N_{B}+N_{G}$. For each top boundary node, approximate the differentiation weights for $\partial / \partial z$ as given in Section 3. This will result in a sparse $N_{B} \times N$ DM (here, called $W$ ). Thus,

$$
\begin{aligned}
\frac{\partial u}{\partial z} & =0 \quad \text { is approximated by } \\
W \underline{u} & =\underline{0} .
\end{aligned}
$$

The function values $\underline{u}$ are organized according to where they are located:

$$
\begin{aligned}
\underline{u}_{I} & : \text { function values at interior nodes } \\
\underline{u}_{B} & : \text { function values at boundary nodes } \\
\underline{u}_{G} & : \text { function values at ghost nodes }
\end{aligned}
$$

Then, the condition $W \underline{u} \approx \underline{0}$ can be written as

$$
W_{I} \underline{u}_{I}+W_{B} \underline{u}_{B}+W_{G} \underline{u}_{G}=\underline{0},
$$

where the matrix $W$ has similarly been split into pieces according to the three different types of nodes:

$$
\begin{gathered}
W_{I}\left(N_{B} \times N_{I}\right): \text { weights applied on interior nodes } \\
W_{B}\left(N_{B} \times N_{B}\right): \text { weights applied on boundary nodes } \\
W_{G}\left(N_{B} \times N_{G}\right): \text { weights applied on ghost nodes }
\end{gathered}
$$

Finally, (4) is used to solve for $\underline{u}_{G}$, the function values at the ghost nodes:

$$
\underline{u}_{G}=-W_{G}^{-1}\left(W_{I} \underline{u}_{I}+W_{B} \underline{u}_{B}\right) .
$$

Once the function values at the ghost node are known, they can be used for the approximation of other derivatives that appear in the governing equations.

\subsection{Hyperviscosity with PHS and polynomials}

When the viscosity of the fluid $\mu$ is small ( such as the case with air $\approx 10^{-5} \mathrm{~m}^{2} / \mathrm{s}$ ), there is essentially no natural diffusion in the governing equations, and high-frequency errors will grow to dominate a numerical solution. To achieve time stability with the RBF-FD method, it has been analyzed and demonstrated for hyperbolic PDEs that adding a relatively small amount of hyperviscosity to the right-hand-side of the governing equations eliminates the contaminating high-frequency noise while keeping the numerically relevant portion of the solution intact $[18,13,4]$.

The hyperviscosity operator takes the form $\gamma \Delta^{k}$, where $k$ is the power the Laplacian and $\gamma$ is a scaling parameter. The integer $k$ controls which frequencies are most affected, with larger values of $k$ giving stronger damping of high frequencies and weaker damping of low frequencies. As has been shown in $[18,13,4]$, for good stability and accuracy, the parameter $\gamma$ is directly proportional 
to the resolution $h$, as well as $k$. Thus, for a square-type domain in $2 \mathrm{D} h \sim 1 / \sqrt{N}$, and $\gamma=c h^{2 k}$, where $c$ is a fine-tuning parameter, generally varying from the values of 0.01 to 1 (for shallow water test cases on the sphere in [13], $c$ there denoted as $\gamma_{c}$ had similar values).

The hyperviscosity operator $\Delta^{k}$ is particularly simple to apply to an odd-powered PHS RBF, regardless of the spatial dimension. The Laplace operator in $d$ dimensions for a radially symmetric function is given by $\Delta=\partial^{2} / \partial r^{2}+((d-1) / r) \partial / \partial r$. Apply this to $\phi(r)=r^{m}$ results in

$$
\Delta\left(\|\mathbf{x}\|^{m}\right)=m[m+(d-2)]\|\mathbf{x}\|^{m-2} .
$$

In other words, applying the Laplace operator to a PHS RBF of degree $m$ gives a new PHS RBF of degree $m-2$. Using the above relationship, one can simply evaluate $\Delta^{k}\left(\|\mathbf{x}\|^{m}\right)$, by applying (5) repeatedly as given in the following example for $d=2$,

$$
\begin{aligned}
\phi(r) & =r^{7} \\
\Delta \phi(r) & =(49) r^{5} \\
\Delta^{2} \phi(r) & =(25)(49) r^{3} \\
\Delta^{3} \phi(r) & =(9)(25)(49) r
\end{aligned}
$$

with the new RBF continuous provided that $(m \geq 2 k+1)$. It should noted that PHS order and degree of polynomials used for spatial discretization need not be that used for hyperviscosity. However, it was found experimentally that the simplest approach was to use up to the same order and degree as that for both. Notice that when finding the weights for the hyperviscosity operator, which is the linear system (3) with $L=\Delta^{k}$, all entries in the right hand side vector pertaining to the polynomial rows will be zero for polynomials of degree less than $k$.

\section{$6 \quad$ Numerical Studies}

The first test case, inviscid transport of a scalar variable in a strongly sheared vortex flow, is a case of pure advection with a known analytical solution, so that the convergence properties of the method can be tested, as well as the effect of stencil size and added polynomial degree. It was originally proposed in [29] and then considered in the context of applying limiters in [3, 40]. The second set of tests is based on the work presented in [43], where a cold descending bubble in a neutrally-stratified atmosphere develops into a traveling density current with the formation of Kelvin-Helmholtz rotors. It is now considered a classic test case in nonhydrostatic atmospheric modeling. The third test [34] (with similar studies in [22]) simulates a rising thermal air bubble and nicely illustrates how instability patterns at the leading edge of it are dependent on the node layout when the dynamic viscosity is that of air.

In all following test cases, $n=37$ node stencils are used for spatial discretization, since both Cartesian and hexagonal layouts have perfectly symmetric stencils at that number, as seen in Appendix A. Although not essential, stencil symmetry is beneficial in that it provides information evenly for approximating an operator at the center of the stencil. Once the equations are spatially discretized, the resulting system of ODEs is time stepped with a 4th-order Runge-Kutta scheme (RK4) (i.e. a method-of-lines (MOL) approach). Although we use always PHS of the form $r^{2 m+1}$, it should be noted that tests with PHS $r^{2 m}=r^{2 m} \log r$ for $m \in \mathbb{N}_{+}$showed no significant differences and could have been used equally well. 


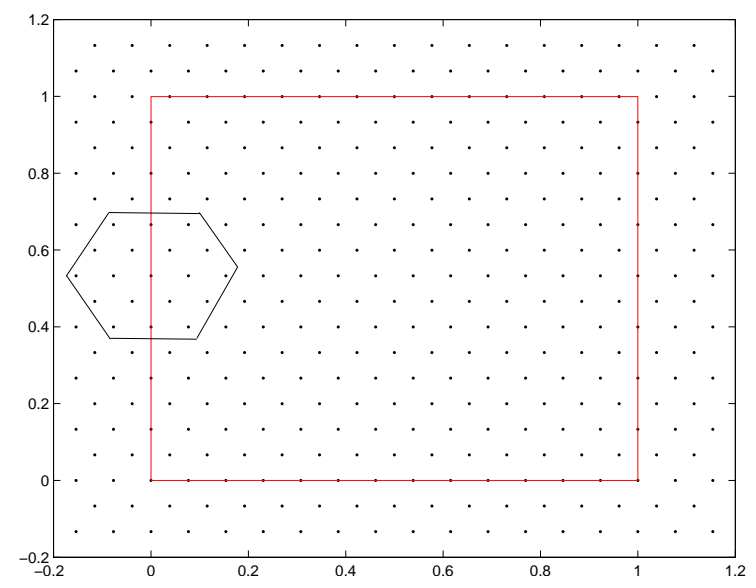

Figure 4: An illustration of a hexagonal 19 node boundary stencil in which the nodes and their associated function values are reflected outside the domain. This setup is used for the advection of a scalar transport test case.

\subsection{Advective transport of a scalar variable}

In this test, a circular scalar field is stretched and deformed into a crescent by vortex-like velocity field that then reverses and returns it back to its original position and shape. The governing equation is defined on $[0,1] \times[0,1]$ in $x$ and $y$ and given by

$$
\frac{\partial \psi}{\partial t}=-\frac{\partial}{\partial x}(u \psi)-\frac{\partial}{\partial y}(v \psi) .
$$

The scalar $\psi$ is advected by the following divergence-free velocity field

$$
u(x, y, t)=u_{\theta}(r, t) \sin \theta, \quad v(x, y, t)=-u_{\theta}(r, t) \cos \theta .
$$

with period $T$, where

$$
u_{\theta}(r, t)=\frac{4 \pi r}{T}\left[1-\cos \left(\frac{2 \pi t}{T}\right) \frac{1-(4 r)^{6}}{1+(4 r)^{6}}\right]
$$

and

$$
r=\sqrt{(x-0.5)^{2}+(y-0.5)^{2}}, \quad \theta=\tan ^{-1}\left(\frac{y-0.5}{x-0.5}\right) .
$$

In order to create a test problem with no boundary effects, the nodes on the interior of the domain near the boundary, as well as the function values associated with them, are simply reflected over the boundary (thus no boundary conditions are needed), forming perfectly symmetric boundary stencils - half of which are then ghost nodes and half interior nodes. The boundary is then time stepped with the rest of the interior of the domain. An example of a symmetric 19 node boundary stencil is given in Figure 4.

The initial condition for $\psi$ is a cosine bell

$$
\left.\psi\right|_{t=0}= \begin{cases}\frac{1+\cos (\pi \tilde{r})}{2} & \tilde{r} \leq 1 \\ 0 & \tilde{r}>1,\end{cases}
$$

where $\tilde{r}=5 \sqrt{(x-0.3)^{2}+(y-0.5)^{2}}$. $\psi$ is advanced in time from $t=0$ until $t=T$ (one period), at which point it should have ideally returned to its original height and position. 


\subsubsection{Accuracy of solution, convergence, and effect of different node layouts}

Figure 5 shows the time evolution for solution (assuming a period of $T=1$ ), with corresponding velocity field, on a hexagonal node layout. The specifications of the resolution, time-step, basis functions and hyperviscosity used are given in the caption of the figure. Although, this is a high resolution case with a total of $N=40401$ nodes in the domain or a node spacing of $h=0.005$, it should be noted that the maximum amplitude of $\psi$ has only increased by $0.07 \%$ and has only gone below zero by $-0.08 \%$, as can be seen in Figure 5a. In fact, when the scalar field is in its state of highest deformation at $t=0.25,0.75$, the error in the maximum amplitude is not more than 0.001. Furthermore, the CFL criterion for an RK4 stability domain dictates a time step of $\Delta t<\Delta x \cdot 2 \sqrt{2} /(\max \mathbf{u})$, where max $\mathbf{u} \approx 18$ for this case as can be calculated from (11). This translates to a $\Delta t<7.8(10)^{-4}$, a factor of about 2.3 larger than the time step taken of $\Delta t=h / 15=0.005 / 15=3.3(10)^{-4}$.

In order to observe the long-time errors in the method, the solution is advanced for 100 periods as shown in the left panel of Figure 6. Even after so many revolutions, the height of the scalar field has decreased only by $4 \%$, with a slight distortion from its circular shape. The right panel of Figure 6 shows a dispersive error pattern with a maximum value of 0.13 . The $\ell_{2}$ and $\ell_{\infty}$ errors are 0.125 and 0.141 , respectively.

For a 37 node stencil, the highest degree polynomials that can be used on all three node sets is fifth degree. Both hexagonal and quasi-uniform node sets can handle sixth degree polynomials but not Cartesian layouts. This is because on such a lattice the nodes approach non-unisolvency, resulting in the column vectors of the polynomial portion of the matrix in (3) becoming linearly dependent. Also, in order to demonstrate that the polynomial degree controls the convergence and not the PHS order, $r^{3}$ PHS are now used instead of $r^{9}$ with up to fifth-degree polynomials on Cartesian, hexagonal and quasi-uniform nodes. Figure 7 illustrates this for three resolutions, $h=0.02$ (2500 points), 0.01 (10000 points), 0.005 (40000 points). For any given resolution, all nodes sets perform roughly the same, both with regard to the minimum and maximum function values and the errors in the $\ell_{2}$ and $\ell_{\infty}$ norm. Comparing the maximum and minimum of the solution for hexagonal nodes on $h=0.005$ between Figures 7 and 5 , it can been seen that using $r^{9}$ gives slightly better accuracy. This phenomena was noted in Section 4. Figure 8 shows the convergence rate corresponding to the cases given in Figure 7. From the discussion in Section 4, the convergence rate should be $O\left(h^{l}\right)$, where $l$ is the highest degree of polynomials used, in this case 5 . Fifthorder convergence is indeed seen for all node sets in Figure 8. Also in this figure, the RBF-FD method is compared to a 5th-order upwind scheme with and without a WENO limiter, both of the latter exhibiting a third-order convergence rate. The reason for the comparison is that this order FD upwinding scheme is the type used in the Weather Research and Forecasting (WRF) Model (http://www.wrf-model.org/). The time steps for both methods are comparable, with less than a $1 \%$ difference.

It should be remembered that this test case was set up to investigate what the numerical results for the proposed RBF-FD method would be under no boundary effects. So, if there is the unusual circumstance that the solution does not interact with any boundaries in a bounded domain and no refinement will be needed, then solving the problem on a Cartesian lattice will give just as good results as hexagonal. Furthermore, the fact that quasi-uniform nodes performed just as well as the other two layouts is of great benefit since it paves the way for the ability to implement local node refinement. 

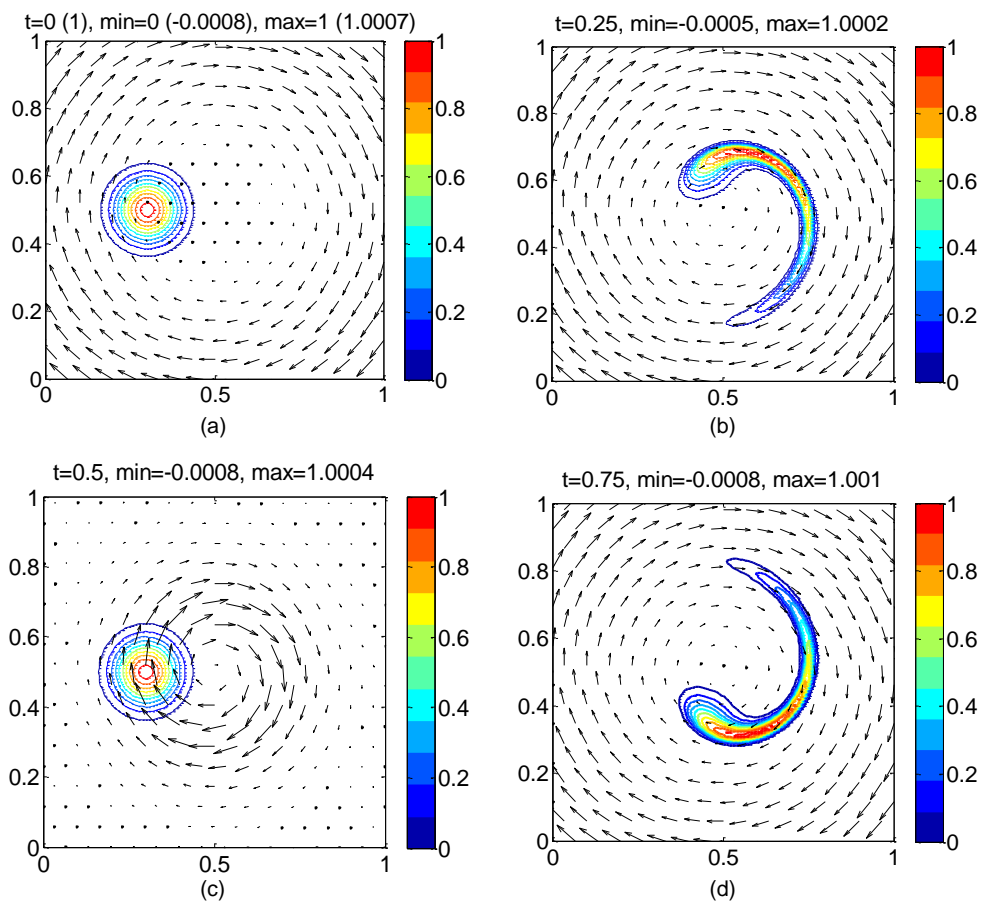

Figure 5: Time series of the solution for $\psi$ at: (a) $t=0(1)$, (b) $t=0.25$, (c) $t=0.5$, (d) $t=0.75$, with the corresponding minimum and maximum values at each time. Contour lines are in intervals of 0.05 . An $n=37$ node stencil with $r^{9}$ PHS and up to $4^{\text {th }}$-order polynomials on a hexagonal node layout of 201 by 201 is used. The time-step is $\mathrm{h} / 15$, where $\Delta x=h=1 / 200=0.005$, as discussed in first paragraph of Section 6.1.1. A hyperviscosity of $0.01 * h^{2 * 4} \Delta^{4}$ is also implemented, as discussed in Section 5.3. 

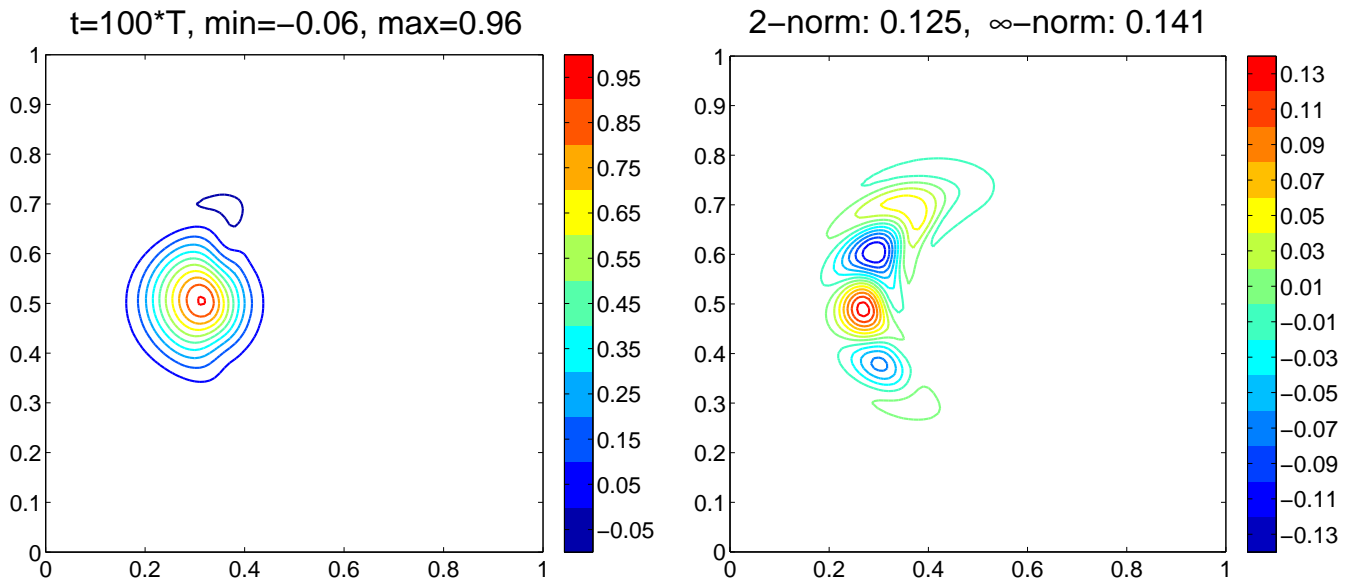

Figure 6: Solution (left panel) and error (right panel) at $t=100 T$ on 40,401 ( $h=0.005$ ) hexagonal nodes using $\phi(r)=r^{9}$ with up to $4^{\text {th }}$ degree polynomials on a 37 -node stencil and $\Delta^{3}$-type hyperviscosity. 
Cartesian
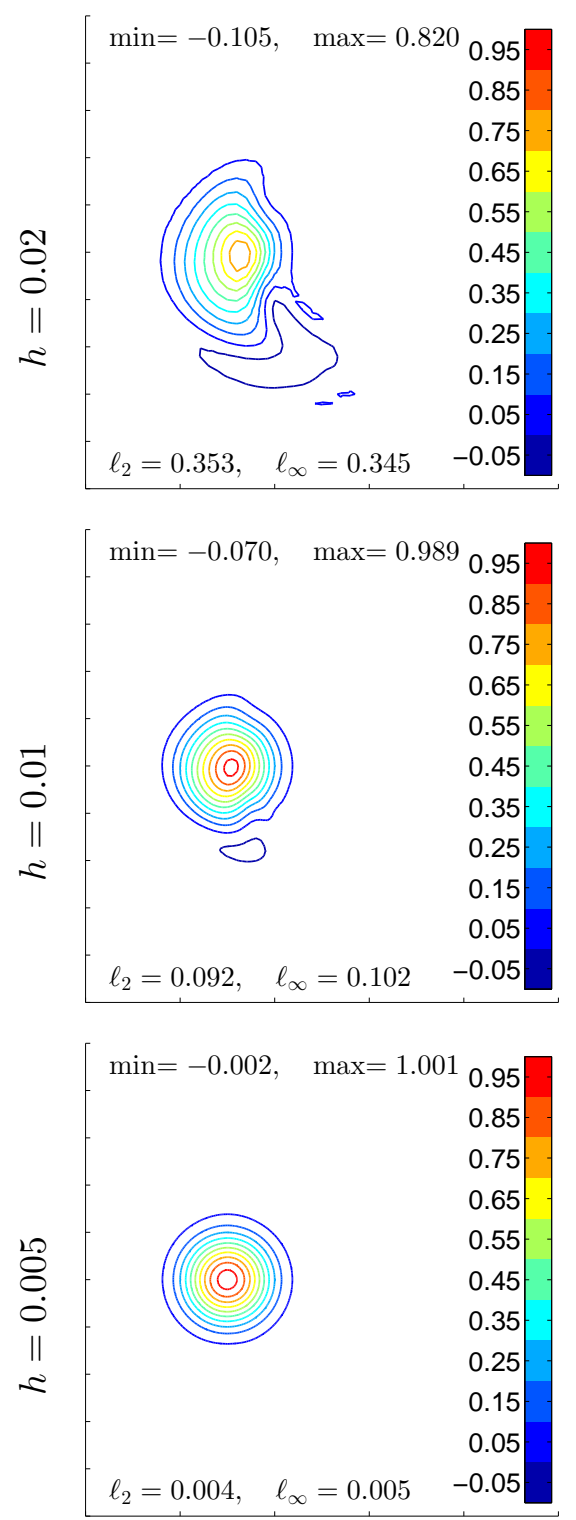

Hexagonal
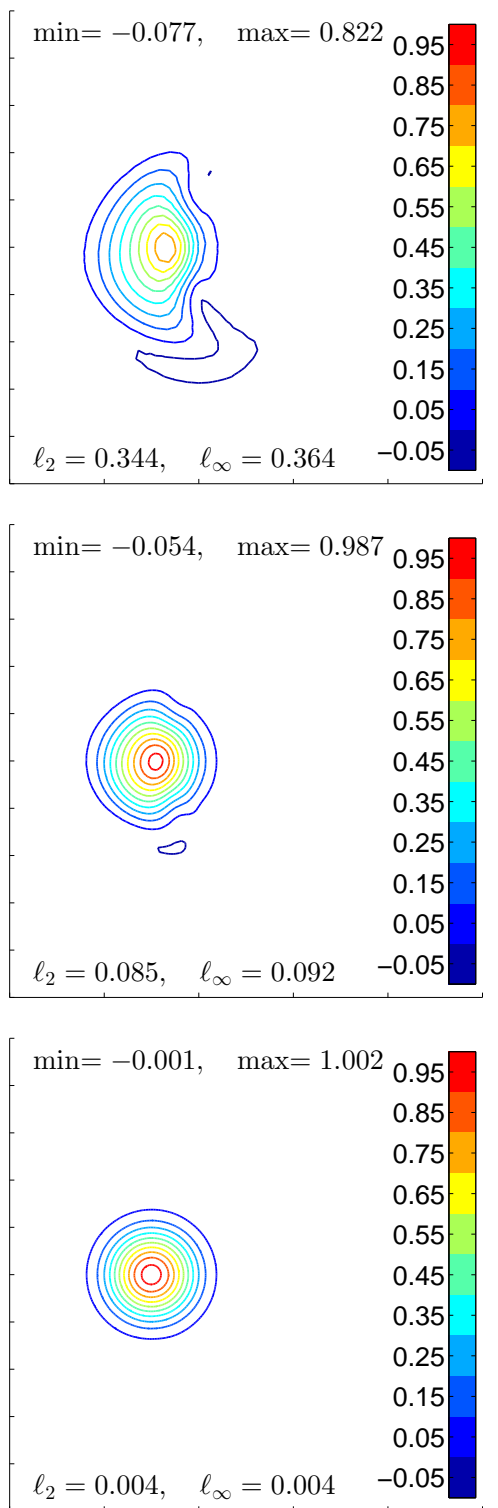

quasi-uniform
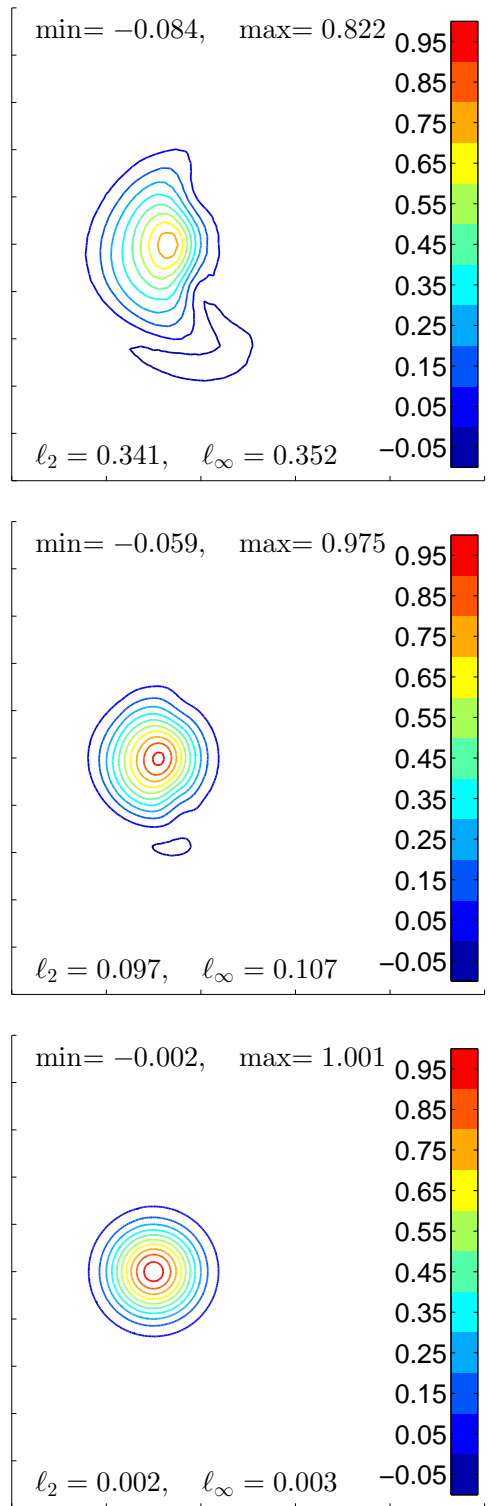

Figure 7: Plots of solutions at $t=T=1$ using the three different types of node-sets. $\phi(r)=r^{3}$, polynomials up to degree 5 on a 37 -node stencil and $\Delta^{3}$-type hyperviscosity were used. The amount of hyperviscosity, $\gamma$, varies between node-sets, but is on the $O(10)^{-12}$ to $O(10)^{-14}$. 


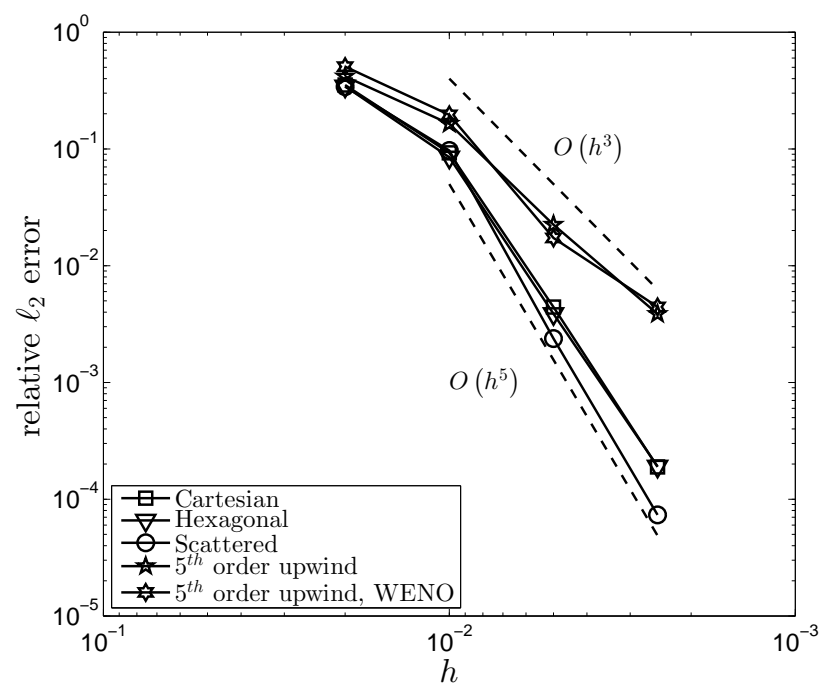

Figure 8: Convergence plots of relative error $\left(\frac{\left\|\psi_{\text {approx }}-\psi_{\text {exact }}\right\|_{2}}{\left\|\psi_{\text {exact }}\right\|_{2}}\right)$ vs. $(h=0.02,0.01,0.005,0.0025)$. In all cases, $\phi(r)=r^{3}$ with polynomials up to degree 5 on a 37 -node stencil and $\Delta^{3}$-type hyperviscosity were used. The error decreases $O\left(h^{5}\right)$, which is expected since up to $5^{\text {th }}$ degree polynomials were included and only first derivatives need to be approximated in the PDE.

\subsubsection{Effect of stencil size and polynomial degree on accuracy}

Since we have the exact solution to this test case at $T=1$, we will now discuss how the polynomial degree used is closely linked to the stencil size. The observations in this section also carry through to the experiments on the Navier-Stokes equations. Table 6.1.2 shows the number of polynomials added, $(l+1)(l+2) / 2$ in $2 \mathrm{D}$, to the PHS $r^{3}$ when doing the calculations in Figure 9 for different stencil sizes. Theoretically, we need one node per polynomial. However, when the number of polynomials approaches the stencil size, the matrix in (3) becomes close to singular (for the same reason that the Vandermonde matrix in the counterpart 1-D case is ill-conditioned). Hence, in Figure 9 for $n=19$, one can only use up to fourth-order polynomials, for $n=37$ - up to sixth-order polynomials and $n=61$ - up to eight-order polynomials. The observations we can make from Figure 9 are:

- For the best accuracy, the number of polynomials terms used should be roughly half the stencil size.

- For a given polynomial degree, stencil size has little impact on the accuracy.

- Going to large stencil sizes $(n=61)$ is not very beneficial.

The interplay between polynomial degree, stencil size, and especially boundaries is still not well understood and is further being pursued in $[12,1]$.

\begin{tabular}{r|lllllll} 
Polynomial (poly.) degree $\ell:$ & 2 & 3 & 4 & 5 & 6 & 7 & 8 \\
\hline Num. of poly. terms up to $\ell:$ & 6 & 10 & 15 & 21 & 28 & 36 & 45
\end{tabular}

Table 2: For a given polynomial degree $\ell$, the number of polynomial terms up to that degree in $2 \mathrm{D}$ that will be augmented. 


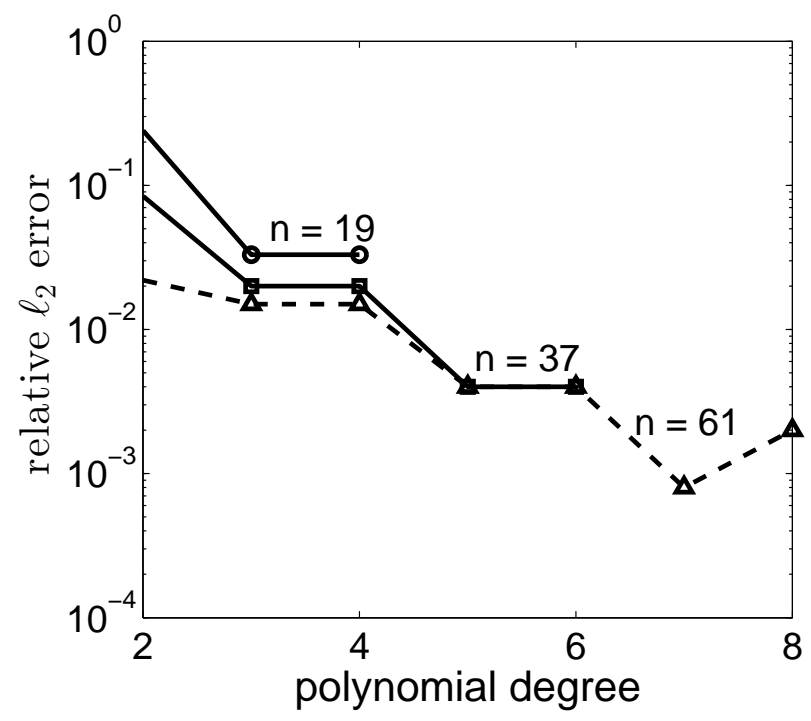

Figure 9: Relative $\ell_{2}$ error of the transport of a scalar test case as a function of polynomials added up to a given degree for different stencil sizes $n$. In all cases, $\phi(r)=r^{3}$ on a hexagonal node layout with $h=0.005$ and $\Delta^{3}$-type hyperviscosity was used.

\subsection{Governing Equations for Navier-Stokes test cases}

In all test cases below, the set of governing equations is the $2 \mathrm{D}$ nonhydrostatic compressible NavierStokes equations at low Mach number, $M \approx 0.1$, in a rectangular or square domain. The equations are given by

$$
\begin{aligned}
& \frac{\partial u}{\partial t}=-u \frac{\partial u}{\partial x}-w \frac{\partial u}{\partial z}-c_{p} \theta \frac{\partial \pi}{\partial x}+\mu \Delta u \\
& \frac{\partial w}{\partial t}=-u \frac{\partial w}{\partial x}-w \frac{\partial w}{\partial z}-c_{p} \theta \frac{\partial \pi}{\partial z}-g+\mu \Delta w \\
& \frac{\partial \theta}{\partial t}=-u \frac{\partial \theta}{\partial x}-w \frac{\partial \theta}{\partial z}+\mu \Delta \theta \\
& \frac{\partial \pi}{\partial t}=-u \frac{\partial \pi}{\partial x}-w \frac{\partial \pi}{\partial z}-\frac{R_{d}}{c_{v}} \pi\left(\frac{\partial u}{\partial x}+\frac{\partial w}{\partial z}\right)
\end{aligned}
$$

where $u$ and $w$ are the velocities in the horizontal and vertical directions, respectively, $\pi=$ $\left(\frac{P}{P_{0}}\right)^{R_{d} / c_{p}}$ is the non-dimensional Exner pressure $\left(P_{0}=1 \times 10^{5} \mathrm{~Pa}\right)$, and $\theta=\frac{T}{\pi}$ is the potential temperature, where $T$ is the current absolute temperature (in K). The constants $c_{p}=1004$ and $c_{v}=717$ are the specific heat at constant pressure and the specific heat at constant volume, respectively, with the gas constant for dry air being $R_{d}=c_{p}-c_{v}=287$. Additional parameters are $g=9.81 \mathrm{~m} / \mathrm{s}^{2}$, the gravitational constant, and $\mu$, the dynamic viscosity. Furthermore, it is assumed that all quantities to be solved for, $[u, w, \theta, \pi]^{T}$, are perturbations $\left({ }^{\prime}\right)$ to a background state $\left(^{-}\right)$that is in hydrostatic balance, i.e. the fluid is initially at rest, $\bar{u}=\bar{w}=0$, and the background Exner pressure is a linear function of height $\mathrm{z}, \frac{d \bar{\pi}}{d z}=-\frac{g}{c_{p} \bar{\theta}}$. Substituting this latter relation into the equations above and writing $\theta=\bar{\theta}+\theta^{\prime}$ and $\pi=\bar{\pi}+\pi^{\prime}$ (where the $\left(^{\prime}\right.$ ) symbol has been dropped 
for reading clarity) yields the governing equations to be used for computation:

$$
\begin{aligned}
\frac{\partial u}{\partial t} & =-u \frac{\partial u}{\partial x}-w \frac{\partial u}{\partial z}-c_{p}(\bar{\theta}+\theta) \frac{\partial \pi}{\partial x}+\mu \Delta u, \\
\frac{\partial w}{\partial t} & =-u \frac{\partial w}{\partial x}-w \frac{\partial w}{\partial z}-c_{p}(\bar{\theta}+\theta) \frac{\partial \pi}{\partial z}+\frac{g \theta}{\bar{\theta}}+\mu \Delta w \\
\frac{\partial \theta}{\partial t} & =-u \frac{\partial \theta}{\partial x}-w \frac{\partial \theta}{\partial z}+\mu \Delta \theta, \\
\frac{\partial \pi}{\partial t} & =-u \frac{\partial \pi}{\partial x}-w\left(\frac{d \bar{\pi}}{d z}+\frac{\partial \pi}{\partial z}\right)-\frac{R_{d}}{c_{v}}(\bar{\pi}+\pi)\left(\frac{\partial u}{\partial x}+\frac{\partial w}{\partial z}\right) .
\end{aligned}
$$

In the following studies, the perturbation notation $\left({ }^{\prime}\right)$ is generally included when reporting results to keep in mind these are perturbation quantities.

\subsection{Numerical Set-up for the NS cases}

For the rest of the paper, the governing equations (12)-(15) are spatially discretized using PHS RBFs, $\phi(r)=r^{7}$, with polynomials up to fourth degree on a stencil-size of $n=37$. The remaining system of first order ODEs in time is solved with RK4. A $\Delta^{3}$-type hyperviscosity is applied in all cases to damp high-frequency modes. The time-step for all test cases as a function of resolution is

\begin{tabular}{r|llllll} 
node-spacing $(h):$ & $800 \mathrm{~m}$ & $400 \mathrm{~m}$ & $200 \mathrm{~m}$ & $100 \mathrm{~m}$ & $50 \mathrm{~m}$ & $25 \mathrm{~m}$ \\
\hline$\Delta t:$ & $2 \mathrm{~s}$ & $1 \mathrm{~s}$ & $\frac{1}{2} \mathrm{~s}$ & $\frac{1}{4} \mathrm{~s}$ & $\frac{1}{8} \mathrm{~s}$ & $\frac{1}{16} \mathrm{~s}$
\end{tabular}

Table 3 below gives the domain size, and number of nodes used as a function of resolution for the numerical studies of the NS test cases.

Table 3: Information regarding the computational domain for each test case. The number of nodes $(N)$ is for hexagonal nodes.

\begin{tabular}{llr}
\hline \hline Test $($ domain size $(x \times z$ in $\mathrm{km}))$ & Resolution $(\mathrm{m})$ & $\approx N$ \\
\hline Straka Density Current $[43]([-25.6,25.6] \times[0,6.4])$ & 800 & 720 \\
& 400 & 2,700 \\
& 200 & 10,000 \\
& 100 & 38,500 \\
& 50 & 152,650 \\
\hline Translating Density Current $[47]([0,36] \times[0,6.4])$ & 800 & 500 \\
& 400 & 1,900 \\
& 200 & 7,040 \\
& 100 & 27,040 \\
& 50 & 107,350 \\
\hline Rising Thermal Bubble $([0,10] \times[0,10])$ & 200 & 2,980 \\
& 100 & 11,760 \\
& 50 & 46,720 \\
& 25 & 185,430 \\
\hline
\end{tabular}




\subsection{Density Current}

In the density current test case [43], a hydrostatic neutral atmosphere is perturbed by a $C^{1}$ bubble in the potential temperature. A mass of cold air falls to the ground and develops three smooth and distinct Kelvin-Helmoltz rotors as it spreads to the sideways. This test has become widely used in weather modeling community for assessing the ability in new numerical schemes to capture the physics in nonhydrostatic fluid flows $[32,21,41,31]$. Figure 10 shows the behavior of the numerical solution in time from $t=0 \mathrm{~s}$ until the final time, $t=900 \mathrm{~s}$.

The computational domain is $[-25.6,25.6] \times[0,6.4] \mathrm{km}^{2}$, and the governing equations (12)-(15) are solved with a viscosity of $\mu=75 \mathrm{~m}^{2} / \mathrm{s}$.

\section{Define $\bar{\theta}$ and $\bar{\pi}$}

Let $\bar{T}(z)=T_{s}-\frac{g}{c_{p}} z$ be the background state for temperature, where $T_{s}=300$ is the temperature at the ground surface in Kelvin. Then, the background states for potential temperature and Exner pressure are given by

$$
\bar{\theta}=T_{s}, \quad \bar{\pi}(z)=\frac{\bar{T}(z)}{\bar{\theta}}=1-\frac{g}{c_{p} T_{s}} z .
$$

\section{Define initial conditions}

The vector of unknowns is initially zero except for the potential temperature.

$$
\left.u\right|_{t=0}=0,\left.\quad w\right|_{t=0}=0,\left.\quad \pi^{\prime}\right|_{t=0}=0 .
$$

The $\left(C^{1}\right)$ initial condition for $\theta^{\prime}$ is derived via a cool cosine bubble in the temperature $T$ defined by

$$
\left.T^{\prime}\right|_{t=0}=\left\{\begin{array}{rr}
-\frac{15}{2}\left\{1+\cos \left[\pi_{c} r(x, z)\right]\right\}, & r(x, z) \leq 1 \\
0, & r(x, z)>1
\end{array}\right.
$$

where $\pi_{c}=3.14159 \ldots$ is the standard trigonometric constant and

$$
r(x, z)=\sqrt{\left(\frac{x-x_{c}}{x_{r}}\right)^{2}+\left(\frac{z-z_{c}}{z_{r}}\right)^{2},} \quad \begin{aligned}
& \left(x_{c}, z_{c}\right)=(0 \mathrm{~km}, 3 \mathrm{~km}), \\
& \left(x_{r}, z_{r}\right)=(4 \mathrm{~km}, 2 \mathrm{~km}) .
\end{aligned}
$$

Then, the initial condition for $\theta^{\prime}$ can be found by dividing by $\bar{\pi}$ :

$$
\left.\theta^{\prime}\right|_{t=0}=[\theta-\bar{\theta}]_{t=0}=\left.\frac{T}{\pi}\right|_{t=0}-T_{s}=\left.\frac{\bar{T}+T^{\prime}}{\bar{\pi}}\right|_{t=0}-T_{s}=\left[\bar{\theta}+\frac{T^{\prime}}{\bar{\pi}}\right]_{t=0}-T_{s}=\frac{\left.T^{\prime}\right|_{t=0}}{\bar{\pi}} .
$$

\section{Define boundary conditions}

The problem is periodic in the $x$ direction with the following conditions on the top and bottom boundaries in $z$ :

$$
w^{\prime}=0, \quad \frac{\partial u^{\prime}}{\partial z}=0, \quad \frac{\partial \theta^{\prime}}{\partial z}=0
$$

These are the only boundary conditions necessary to solve the governing equations. However, enforcing the vertical momentum equation (13) on the top and bottom boundaries and assuming 
that perturbation in the pressure gradient balances the perturbation in the potential temperature leads to the following condition for $\pi^{\prime}$,

$$
\frac{\partial \pi^{\prime}}{\partial z}=\frac{g \theta^{\prime}}{c_{p} \bar{\theta}\left(\bar{\theta}+\theta^{\prime}\right)}
$$

Furthermore, since the dynamic viscosity $\mu$ for air is $\approx 10^{-5}$, a good approximation on the top and bottom boundaries is $\Delta w^{\prime}=0$ or $\partial^{2} w^{\prime} / \partial z^{2}=0$ since $w=0$ on these boundaries. While these two extra boundary conditions on $\pi^{\prime}$ and $w^{\prime}$ are not required, they allow for the use of ghost nodes in all four variables. In summary, the lateral boundaries are periodic, and the complete set of boundary conditions enforced on the top and bottom boundaries is:

$$
w=\frac{\partial^{2} w}{\partial z^{2}}=\frac{\partial u}{\partial z}=\frac{\partial \theta^{\prime}}{\partial z}=0, \quad \frac{\partial \pi^{\prime}}{\partial z}=\frac{g \theta^{\prime}}{c_{p} \bar{\theta}\left(\bar{\theta}+\theta^{\prime}\right)} .
$$
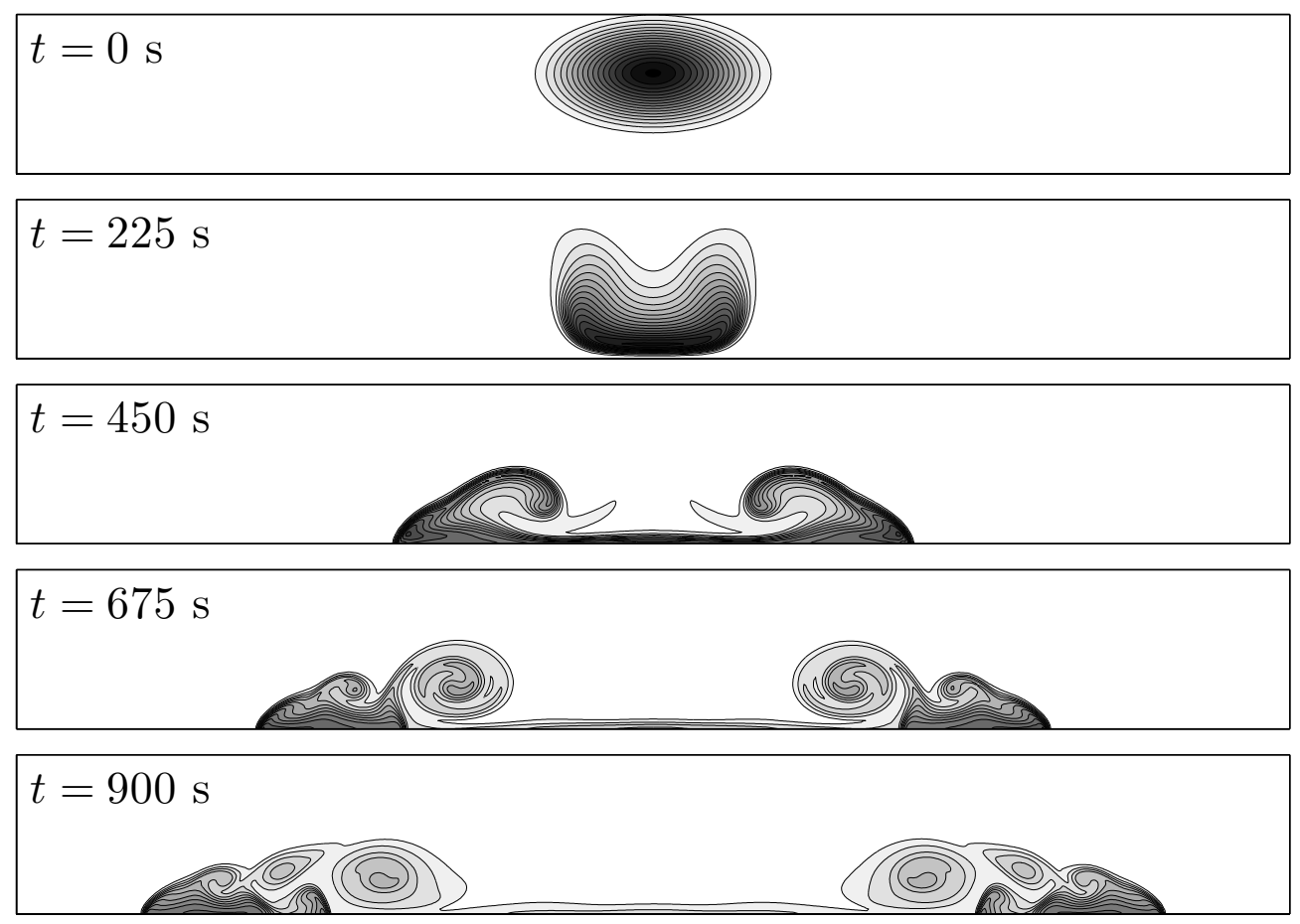

Figure 10: Time evolution of the potential temperature perturbation $\theta^{\prime}$ for the density current test case with $\mu=75 \mathrm{~m}^{2} / \mathrm{s}$ at a $100 \mathrm{~m}$ resolution on hexagonal nodes. 


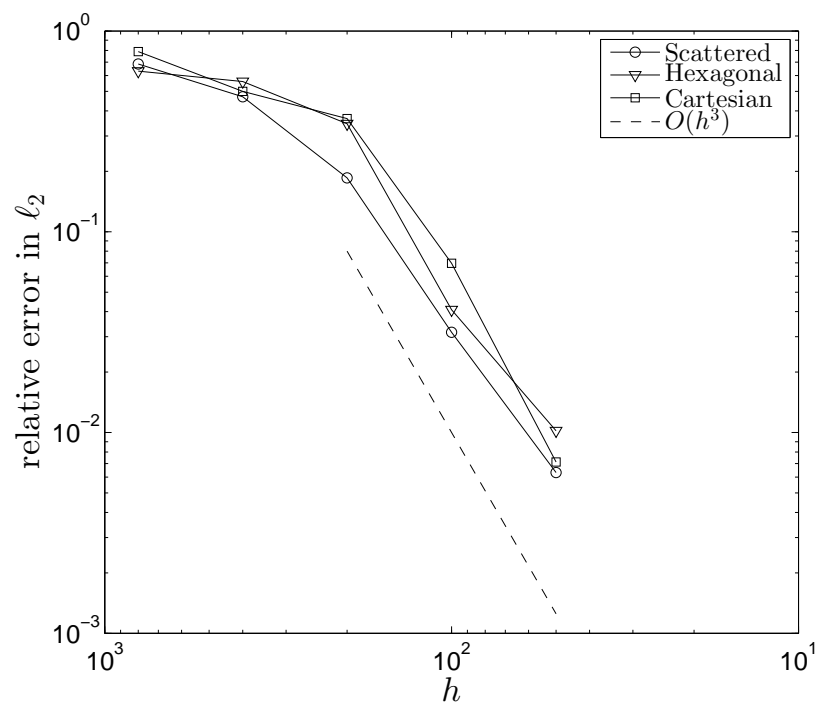

Figure 11: Convergence behavior for $\theta^{\prime}$ in the Straka density current test case with $\mu=75 \mathrm{~m}^{2} / \mathrm{s}$. The $h=800 \mathrm{~m}, 400 \mathrm{~m}, 200 \mathrm{~m}, 100 \mathrm{~m}$, and $50 \mathrm{~m}$ errors were calculated using the $25 \mathrm{~m}$ RBF-FD reference solution.
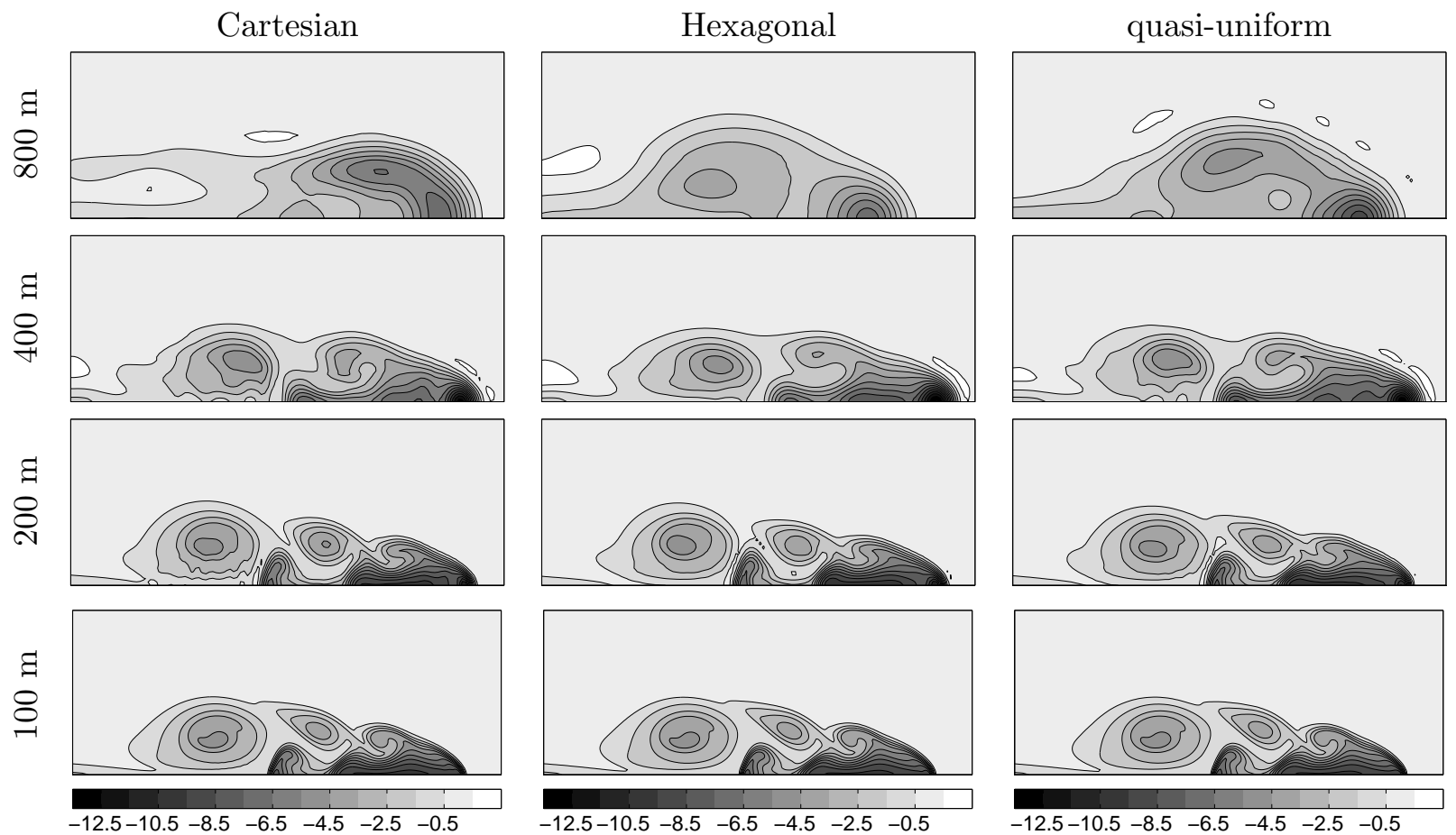

Figure 12: The solution at the final time for the density current test case solved on different node layouts for resolutions $800 \mathrm{~m}, 400 \mathrm{~m}, 200 \mathrm{~m}, 100 \mathrm{~m}$ (for each resolution, the three cases have virtually the same number of nodes). Only half the solution is shown to enlarge details. Contours for the density current begin at $-0.5 \mathrm{~K}$ and are in intervals of $1 \mathrm{~K}$. The white areas are enclosed by a contour of $0.5 \mathrm{~K}$. 
In Figure 11, the $\ell_{2}$ error is approximately the same regardless of the node layout with a thirdorder convergence rate for node resolutions $200 \mathrm{~m}$ and less. However, convergence rates do not illustrate how the physics is being resolved with regard to where the data is sampled (i.e. in terms of the node layout). As a result, in Figure 12, the solution of the density current test case is given on the three different node layouts discussed in Section 5 for four different resolutions, varying from $800 \mathrm{~m}$ to $100 \mathrm{~m}$. In the highest resolution displays $(100 \mathrm{~m})$, although all node layouts seemed to have converged to the same solution, differences can be noted in Table 4, where a $25 \mathrm{~m}$ test run on hexagonal nodes is used as a reference solution. Results from a $50 \mathrm{~m}$ test run on the 3 different node sets are also given in the table. By $100 \mathrm{~m}$ on hexagonal and quasi-uniform nodes the minimum $\theta^{\prime}$ has converged, while the maximum $\theta^{\prime}$ is still slightly overshooting zero. Similar error percentages can be found in $w^{\prime}$ and in the front location, with Cartesian nodes performing the worst. Notice that the front for the $100 \mathrm{~m}$ Cartesian is at the same location as achieved by a $200 \mathrm{~m}$ hexagonal layout.

At $400 \mathrm{~m}$ and $800 \mathrm{~m}$, values in the table will be far off the converged $25 \mathrm{~m}$ solution. Instead, noting physical features of the solution in Figure 12, such as 1) at what resolution do the rotors begin to form, their shape and where, and 2) how much cold air has been entrenched in each rotor, will give a better idea of the capability of the node layout to capture the physics. The following observations can be made:

1. At $800 \mathrm{~m}$ : The hexagonal and quasi-uniform node solutions give more clear evidence of the first (largest) rotor being formed. The $-3.5 \mathrm{~K}$ contour in the hexagonal case (circular inner most contour in the first rotor) is even close to its final position if compared to the $100 \mathrm{~m}$ case. Although the first rotor for the quasi-uniform case is not as nicely formed, it has entrenched more cold air, already having the $-4.5 \mathrm{~K}$ contour (teardrop shape) that appears in the $100 \mathrm{~m}$ solution. In comparison, the $800 \mathrm{~m}$ Cartesian has barely any rotor formation and is much more wildly oscillatory, which is also noted by the fact that the maximum $\theta^{\prime}$ is $2.43 \mathrm{~K}$, at least 1.3K larger than for the other node layouts. See Table 4. Both hexagonal and quasi-uniform nodes undershoot the correct position while Cartesian overshoot it.

2. At 400m: Oscillations due to boundary error effects especially in the first rotor are very pronounced on the Cartesian layout; this carries over even to the $200 \mathrm{~m}$ resolution for this node case. For quasi-uniform nodes there are minor oscillations in the solution. For the hexagonal case, barely any are evident. Formation of the second rotor has the nicest intact shape with the least amount of oscillation in both the hexagonal and quasi-uniform, with the latter having entrenched a slightly larger amount of cold air (notice the size of the $-3.5 \mathrm{~K}$ contour teardrop-shaped area in the second rotor of the quasi-uniform case).

The differences between the columns of subplots reflect only the intrinsic resolution capabilities of the different node layouts for capturing the physics. The traditional Cartesian choice is the least effective one. At every resolution level, the hexagonal and quasi-uniform choices give better accuracy than the Cartesian one. The advantage of generalizing from hexagonal to quasi-uniform nodes, is that it then becomes easy to implement spatially variable node densities, i.e. to do local refinement in select critical areas. It is very important to note that this major increase in geometric flexibility (from hexagonal to quasi-uniform) hardly has any negative effect at all on the accuracy that is achieved, nor on the algorithmic complexity of the code. 
Table 4: Resolution $(h)$, minimum and maximum values for $\theta^{\prime}$ and $w$, and front location at various resolutions for the density current test case with $\mu=75 \mathrm{~m}^{2} / \mathrm{s}$. The front location was determined by the $-0.5 \mathrm{~K}$ contour line.

\begin{tabular}{c|c|c|c|c|c|c} 
& $\mathrm{h}(\mathrm{m})$ & $\min \left\{\theta^{\prime}\right\}$ & $\max \left\{\theta^{\prime}\right\}$ & $\min \left\{w^{\prime}\right\}$ & $\max \left\{w^{\prime}\right\}$ & front $(\mathrm{m})$ \\
\hline \hline Cartesian & 800 & -7.74 & 2.43 & -9.19 & 11.00 & 16,079 \\
& 400 & -13.45 & 1.10 & -15.21 & 16.36 & 16,013 \\
& 200 & -12.15 & 0.57 & -16.59 & 17.49 & 15,799 \\
& 100 & -9.84 & 0.27 & -16.14 & 13.45 & 15,500 \\
& 50 & -9.71 & 0.04 & -15.96 & 12.86 & 15,424 \\
\hline quasi-uniform & 800 & -8.60 & 1.11 & -10.11 & 10.05 & 15,477 \\
& 400 & -12.03 & 1.13 & -13.26 & 12.79 & 15,747 \\
& 200 & -10.40 & 0.42 & -15.90 & 13.60 & 15,597 \\
& 100 & -9.70 & 0.21 & -16.00 & 13.12 & 15,447 \\
& 50 & -9.70 & 0.02 & -15.95 & 12.87 & 15,422 \\
\hline Hexagonal & 800 & -6.90 & 1.00 & -11.53 & 9.61 & 15,101 \\
& 400 & -13.38 & 0.98 & -12.93 & 10.11 & 15,721 \\
& 200 & -11.42 & 0.44 & -15.90 & 14.34 & 15,501 \\
& 100 & -9.70 & 0.20 & -15.90 & 12.96 & 15,444 \\
& 50 & -9.70 & 0.01 & -15.93 & 12.90 & 15,420 \\
\hline Reference & 25 & -9.70 & 0.00 & -15.93 & 12.90 & 15,418
\end{tabular}

\subsubsection{Low-Viscosity Density Current $\mu=2 \times 10^{-5} \mathrm{~m}^{2} / \mathbf{s}$}

Here, the density current test case is repeated, except with the dynamic viscosity $\mu$ set to that of air. The purpose of this test case is to show that one can stably time step the RBF-FD method in a completely turbulent regime. The same amount of hyperviscosity as well as the same time step are used in this test case as in the one with $\mu=75 \mathrm{~m}^{2} / \mathrm{s}$. Even in this low-viscosity regime, time stability is governed solely by the fact that the time step could not exceed the speed of sound in air. This is the ideal situation since explicit time-steppers can not exceed the CFL condition.

At such low viscosity, the solution enters the turbulent regime. In such regimes, there is no convergence to any solution as energy cascades to smaller and smaller scales, eventually entering the sub-grid scale domain. Nevertheless, it is interesting to observe whether the model remains stable in this regime. Figure 13 shows the solution at $100 \mathrm{~m}, 50 \mathrm{~m}$ and $25 \mathrm{~m}$ resolutions on the three different node layouts. For any given resolution the solution looks completely different depending on the node layout. This is to be expected as changing the node layout in practically the absence of explicit viscosity is equivalent to introducing slight perturbations in the solution. A more robust illustration of this will be given in the test case of a rising thermal bubble, Section 6.5. 

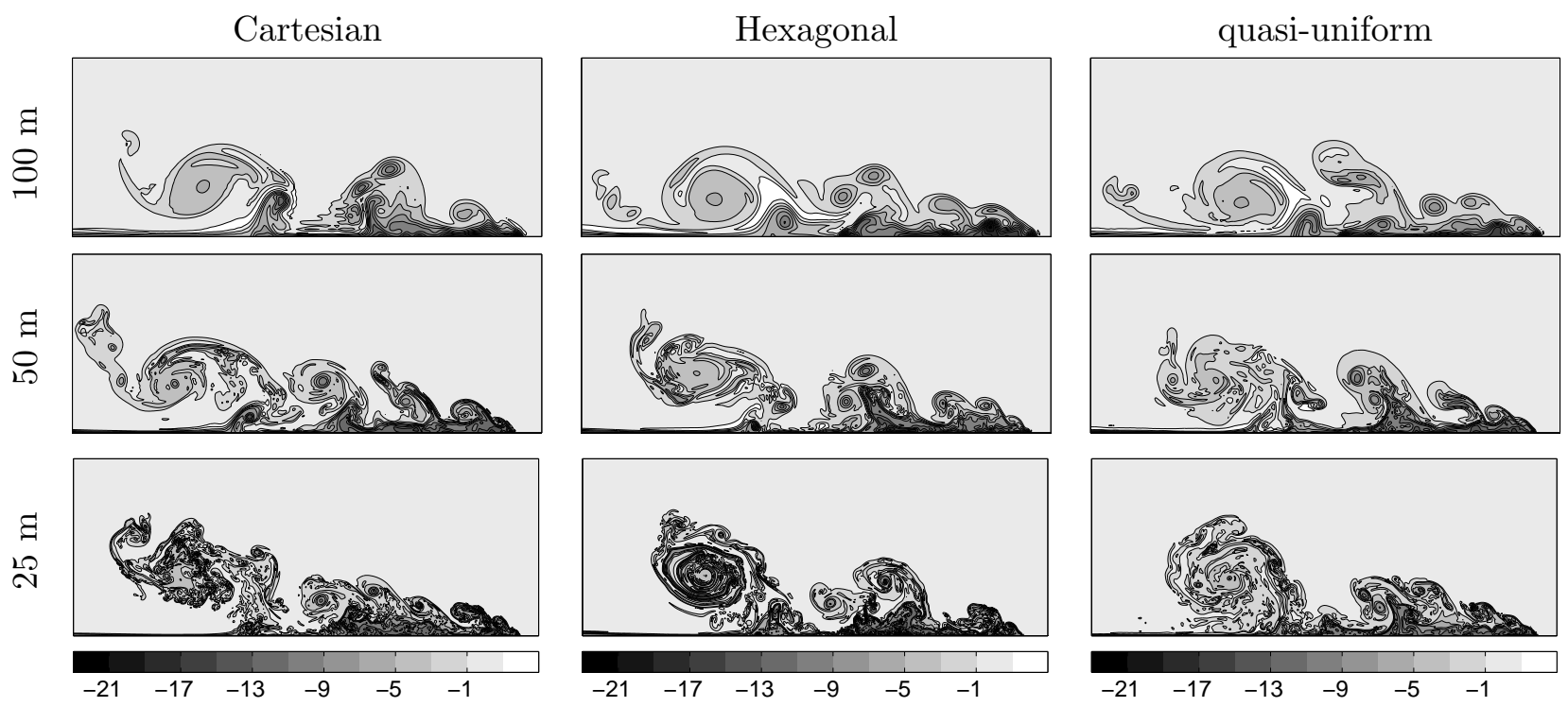

Figure 13: Potential temperature perturbation $\theta^{\prime}$ with $\mu=2 \times 10^{-5} \mathrm{~m}^{2} / \mathrm{s}$ using $100 \mathrm{~m}, 50 \mathrm{~m}$, and $25 \mathrm{~m}$ nodes at $t=900 \mathrm{~s}$.

\subsubsection{Translating Density Current, $\mu=75 \mathrm{~m}^{2} / \mathrm{s}$}

This test is the same as in Section 6.4, except that the domain is now $[0,36] \times[0,6.4] \mathrm{km}^{2}$ and there is a horizontal background wind of $\bar{u}=20 \mathrm{~m} / \mathrm{s}$. The size of the domain is set up so that at $t=900 \mathrm{~s}$ the two "halves" of the solution should be symmetric about $x=18 \mathrm{~km}$. The introduction of a background mean flow introduces a large difference in the movement of each half of the solution. The right portion of the outflow has horizontal velocities $\sim 50 \mathrm{~m} / \mathrm{s}$, while the left portion has velocities $\sim 10 \mathrm{~m} / \mathrm{s}$. As a result, it tests the ability of the scheme to translate the features of the solution at the correct speeds and to generate the correct rotor structures that arise from the local shearing instabilities. For this case, only Cartesian and hexagonal nodes are considered, as the case tests the degree to which symmetry is broken in the two halves of the solution at the final time. Figure 14 illustrates the time series of the potential temperature $\theta^{\prime}$ field, showing how the right part of the solution is advected through the right side of the domain, with the front locations facing one another at 900s (instead of facing the lateral boundaries as in the previous test case).

In Figure 15, the two halves of the solution are compared about the line of symmetry $(18 \mathrm{~km})$ for Cartesian and hexagonal nodes from $800 \mathrm{~m}$ to $100 \mathrm{~m}$ resolutions. The general observations that can be seen are:

1. The $800 \mathrm{~m}$ hexagonal node layout performs highly superior to the Cartesian both in terms of symmetry between the two sides, intactness of the large rotor, and its relative location when compared to the $100 \mathrm{~m}$ case.

2. At both the $400 \mathrm{~m}$ and $200 \mathrm{~m}$ Cartesian case, the left half of the solution that has been advected through the right boundary displays a significant amount of Runge phenomena ('wiggles' in the contour lines near the boundary at $200 \mathrm{~m}$ and severe distortion of the primary and secondary rotor at $400 \mathrm{~m}$ ). This is not the case for hexagonal nodes, which at $400 \mathrm{~m}$ and $200 \mathrm{~m}$, shows relatively nice symmetry between the two sides. 
3. At $100 \mathrm{~m}$, there is no distinction between the two node sets.

The actual front locations, in terms of their distance from the $18 \mathrm{~km}$ mark, are given in Table 5 . Note that the front on the right is farther from the line of symmetry (18km mark) than the one that has been advected through the boundary for the resolutions $800 \mathrm{~m}$ to $100 \mathrm{~m}$. At $50 \mathrm{~m}$, both the Cartesian and hexagonal case is symmetric about the $18 \mathrm{~km}$ mark. However the distance from the front to the line of symmetry varies between the two cases, $2586 \mathrm{~m}$ versus $2595 \mathrm{~m}$, respectively.

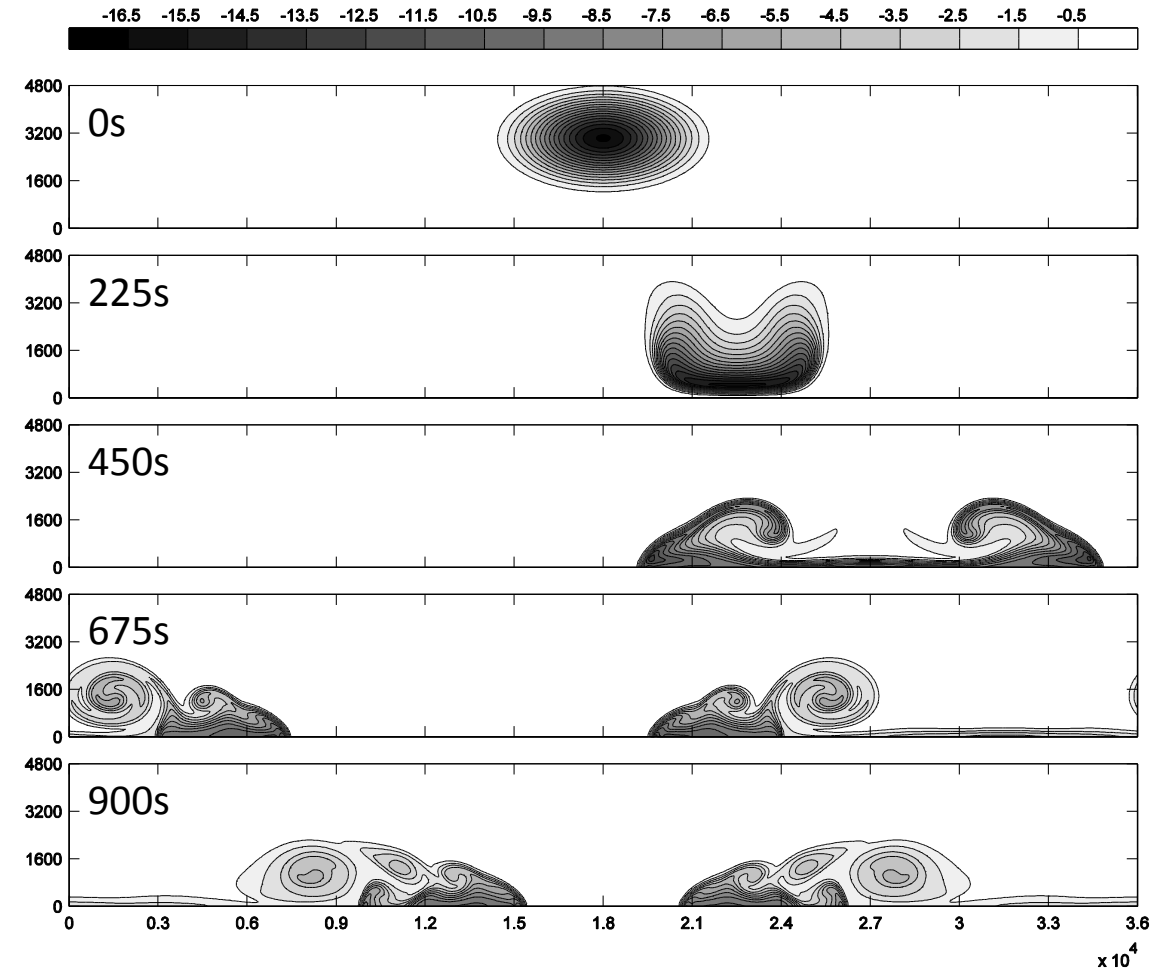

Figure 14: Time evolution of the potential temperature $\theta^{\prime}$ for the translating density current test case. Snapshots were generated using the $100 \mathrm{~m}$ RBF-FD solution on hexagonal nodes. 

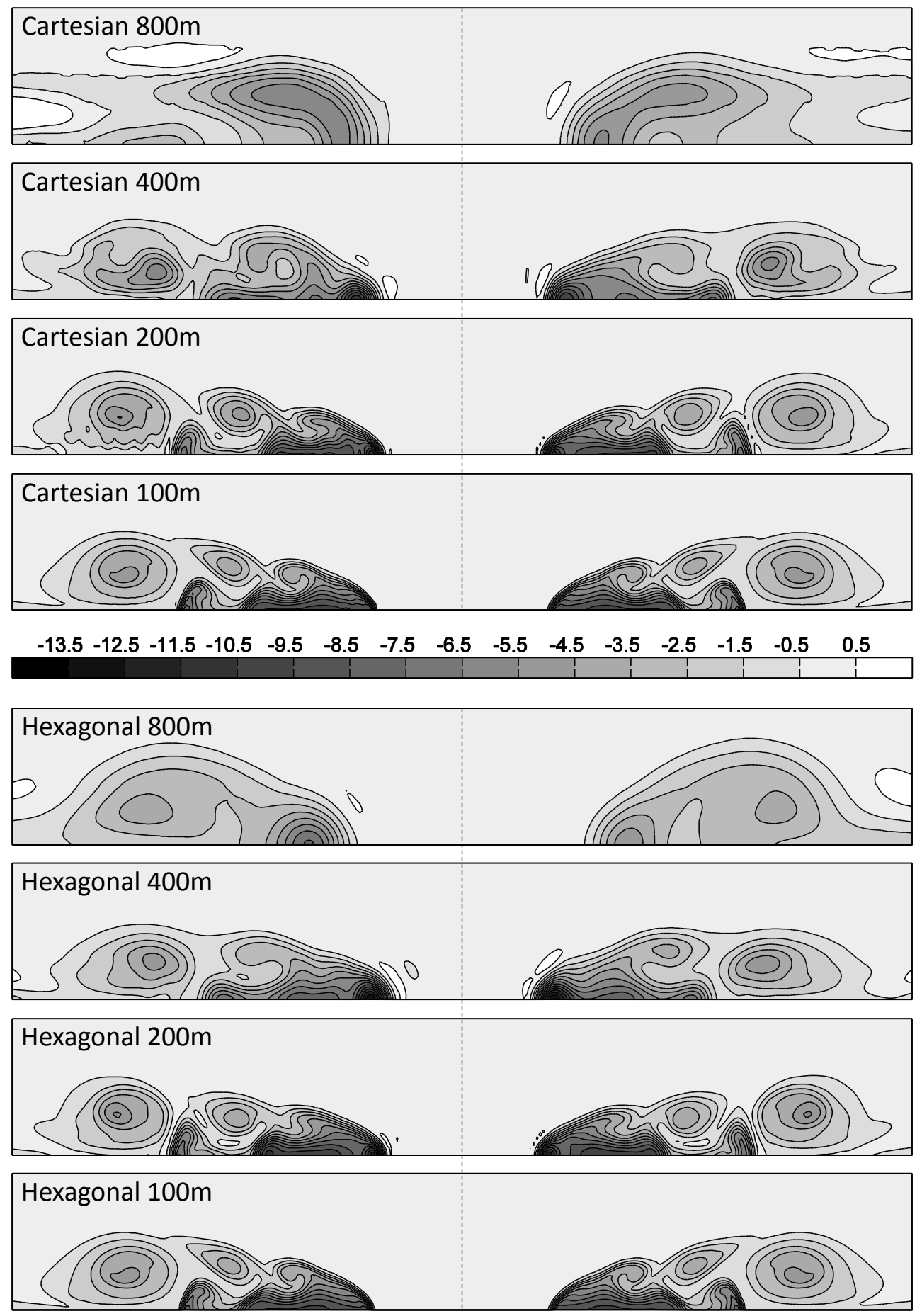

Figure 15: Potential temperature perturbation $\theta^{\prime}$ for the translating density current at $t=900 \mathrm{~s}$ for Cartesian and hexagonal nodes at given resolutions. The dashed line is the $x=18 \mathrm{~km}$ mark about which the two halves should be symmetric. 
Table 5: Left and right front locations for the translating density current test case 6.4 .2 as given by the distance from the $-0.5 \mathrm{~K}$ contour line to the center of the domain, $x=18 \mathrm{~km}$. All values are calculated at the final time, $t=900 \mathrm{~s}$. A $25 \mathrm{~m}$ reference solution is given for the hexagonal nodes.

\begin{tabular}{c|c|c|c} 
& \multicolumn{3}{|c}{} \\
& $\mathrm{h}$ & left front $(\mathrm{m})$ & right front $(\mathrm{m})$ \\
\hline \hline Cartesian & 800 & 3,065 & 3,215 \\
& 400 & 1,915 & 2,085 \\
& 200 & 2,098 & 2,205 \\
& 100 & 2,487 & 2,512 \\
& 50 & 2,586 & 2,586 \\
\hline Hexagonal & 800 & 3,410 & 3,575 \\
& 400 & 1,956 & 2,046 \\
& 200 & 2,165 & 2,260 \\
& 100 & 2,555 & 2,580 \\
& 50 & 2,595 & 2,595 \\
\hline Reference & 25 & 2,595 & 2,595
\end{tabular}

\subsection{Rising Thermal Bubble}

With this last test case, the paper comes full circle in that the presented numerical method is tested on a problem with sharp gradients and very little boundary interaction as in the advective transport of a scalar variable, but is modeled by the same 2D nonhydrostatic compressible NavierStokes equations as in the density current tests. The only difference is the initial condition is given by a $C^{0}$ cone-shaped perturbation. The bubble is warmer than the surrounding atmosphere and thus rises toward the top boundary. However, the time interval and domain size are chosen so that the bubble never interacts with the boundaries.

There are two variations for this test case:

1. $\mu=10 \mathrm{~m}^{2} / \mathrm{s}$ : By adding a small amount of explicit viscosity, the convergence behavior of the solution can be studied.

2. $\mu=2 \times 10^{-5} \mathrm{~m}^{2} / \mathrm{s}$ : At such low viscosity, the bubble is in a turbulent regime, and the behavior of the true solution is unknown. The purpose of the test is to see if the RBF-FD method can give reasonable results when the initial condition is not even continuously differentiable as well as observe how the instability pattern at the leading edge of thermal bubble changes with the node layout.

\subsubsection{Case $\mu=10 \mathrm{~m}^{2} / \mathrm{s}$}

The computational domain is $[0,10] \times[0,10] \mathrm{km}^{2}$. The hydrostatic background states are defined by $\bar{\theta}=T_{s}$ and $\bar{\pi}(z)=1-\frac{g}{c_{p} T_{s}} z$, with $T_{s}=300 \mathrm{~K}$ being the surface temperature. The horizontal and vertical velocities and the Exner pressure perturbation $\left(\pi^{\prime}\right)$ are initially zero, while the potential temperature perturbation is prescribed as a warm cone-shaped "bubble" with a jump in the first derivative $\left(C^{0}\right)$ :

$$
\left.\theta^{\prime}\right|_{t=0}=2 \max \{0,1-r(x, z) / R\} .
$$


Here, $R=1.5 \mathrm{~km}$ is the radius of the bubble, and

$$
r(x, z)=\sqrt{\left(x-x_{c}\right)^{2}+\left(z-z_{c}\right)^{2}}, \quad\left(x_{c}, z_{c}\right)=(5 \mathrm{~km}, 3 \mathrm{~km}) .
$$

The same boundary conditions as in the density current problem are enforced on the top and bottom boundaries

$$
w=\frac{\partial^{2} w}{\partial z^{2}}=\frac{\partial u}{\partial z}=\frac{\partial \theta^{\prime}}{\partial z}=0, \quad \frac{\partial \pi^{\prime}}{\partial z}=\frac{g \theta^{\prime}}{c_{p} \bar{\theta}\left(\bar{\theta}+\theta^{\prime}\right)} .
$$

The lateral boundary conditions are given by

$$
u=\frac{\partial^{2} u}{\partial x^{2}}=\frac{\partial w}{\partial x}=\frac{\partial \theta^{\prime}}{\partial x}=\frac{\partial \pi^{\prime}}{\partial x}=0 .
$$

Figure 16 shows the time series of the solution for a $25 \mathrm{~m}$ resolution $(N=185,730)$ on hexagonal nodes using $r^{7}$ with up to 4 th-order polynomials and a $\Delta^{3}$-type hyperviscosity. The main purpose of this test is to make sure that, under refinement, all node layouts converge to the same solution (as this will not be the case in the next variation of the test) and to see if the convergence rate follows the predictions of Section 4, even with a $C^{0}$ initial condition. Figure 17 shows the final solution for the three different node layouts from a resolution of $200 \mathrm{~m}$ to $25 \mathrm{~m}$. At $25 \mathrm{~m}$ resolution, all solutions are visually identical. At $100 \mathrm{~m}$, Cartesian and hexagonal nodes are more similar with quasi-uniform nodes having more incongruities at the leading edge of the rising bubble. A possible reason for this could be symmetry-breaking associated with quasi-uniform node layouts that would affect areas of large shear.

In terms of convergence, Figure 18 shows that even though the initial condition is $C^{0}$, the method does achieve 4 th order convergence as predicted when using up to fourth degree polynomials. All nodes sets converge at fourth order under refinement, with Cartesian giving the best accuracy. Table 6 shows to what degree the solution has converged in terms of how high the bubble should have risen, $\theta^{\prime}$, and $w^{\prime}$. When comparing against the $12.5 \mathrm{~m}$ hexagonal node reference solution, most variables have converged (or are very close to being converged) by $50 \mathrm{~m}$ for all node layouts.
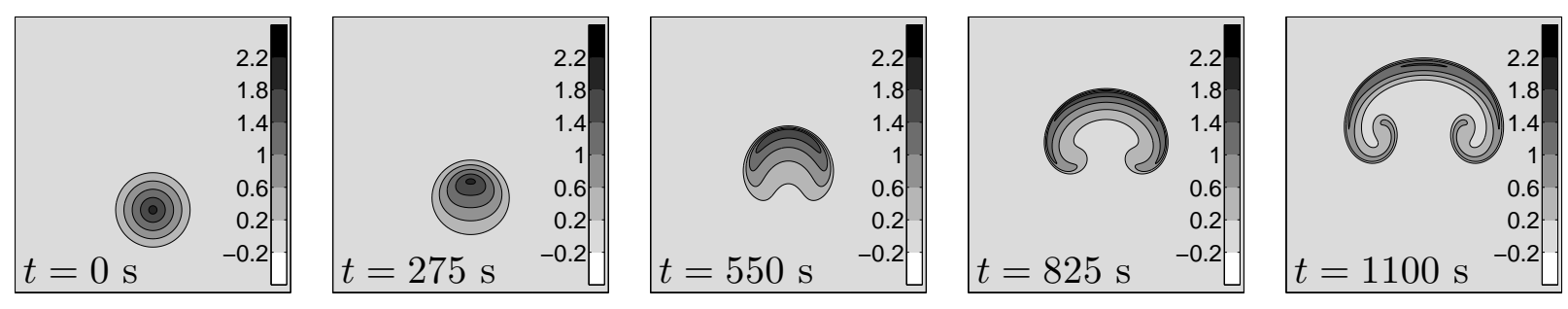

Figure 16: Time evolution of the potential temperature $\theta^{\prime}$ for the $\mu=10 \mathrm{~m}^{2} / \mathrm{s}$ rising thermal bubble. Snapshots were generated using the $25 \mathrm{~m}$ RBF-FD solution on hexagonal nodes. 

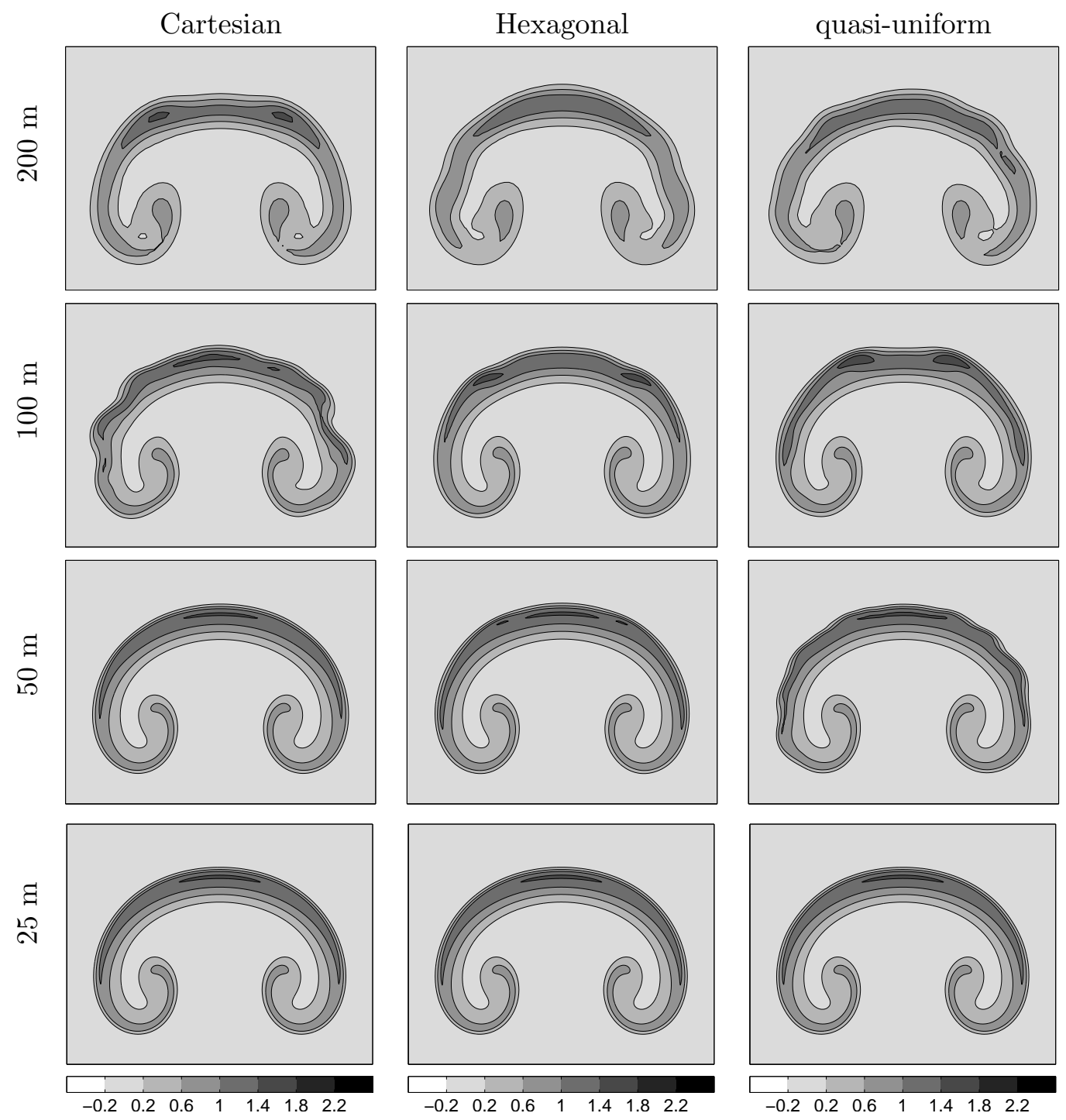

Figure 17: Numerical solutions for the rising thermal bubble with $\mu=10 \mathrm{~m}^{2} / \mathrm{s}$ (6.5.1) on the three different types of node distributions at various resolutions, shown at the final simulation time, $t=1100 \mathrm{~s}$. 


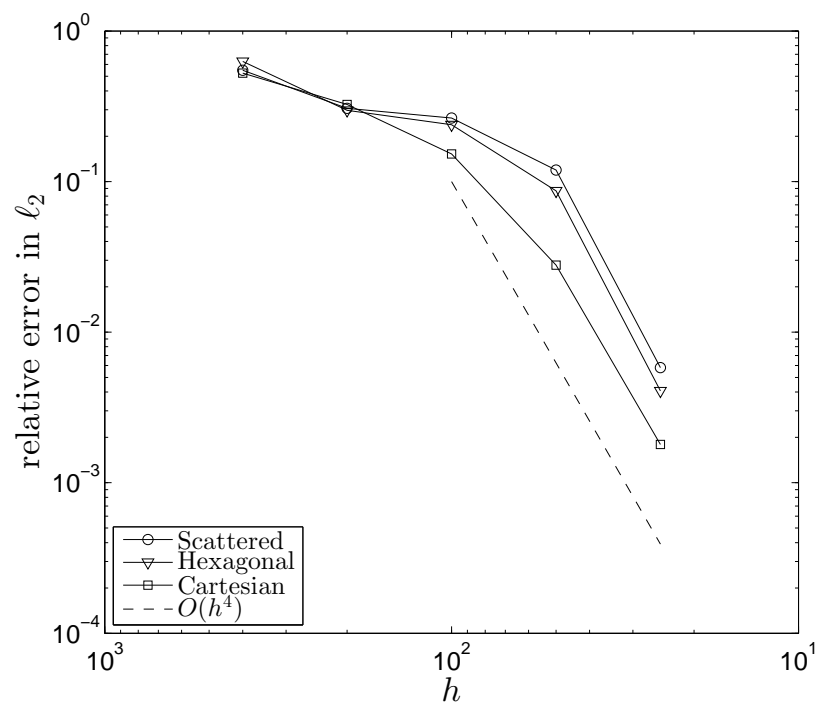

Figure 18: Convergence behavior for $\theta^{\prime}$ in the rising thermal bubble test case 6.5 .1 . The $h=400 \mathrm{~m}$, $200 \mathrm{~m}, 100 \mathrm{~m}, 50 \mathrm{~m}$, and $25 \mathrm{~m}$ errors were calculated using the $12.5 \mathrm{~m}$ RBF-FD reference solution.

Table 6: Resolution $(h)$, minimum and maximum values for $\theta^{\prime}$ and $w^{\prime}$, and bubble height at various resolutions for the rising thermal bubble 6.5.1. The bubble height was determined by the intersection of the $0.1 \mathrm{~K}$ contour and the line $x=5 \mathrm{~km}$.

\begin{tabular}{c|c|c|c|c|c|c} 
& $\mathrm{h}(\mathrm{m})$ & $\min \left\{\theta^{\prime}\right\}$ & $\max \left\{\theta^{\prime}\right\}$ & $\min \left\{w^{\prime}\right\}$ & $\max \left\{w^{\prime}\right\}$ & bubble height $(\mathrm{m})$ \\
\hline \hline Cartesian & 200 & -0.11 & 1.46 & -7.56 & 11.06 & 8,467 \\
& 100 & -0.08 & 1.53 & -7.93 & 11.34 & 8,539 \\
& 50 & -0.02 & 1.43 & -7.87 & 11.43 & 8,534 \\
& 25 & 0.00 & 1.43 & -7.74 & 11.43 & 8,535 \\
\hline Hexagonal & 200 & -0.11 & 1.36 & -7.67 & 11.12 & 8,686 \\
& 100 & -0.09 & 1.65 & -8.05 & 11.49 & 8,527 \\
& 50 & -0.02 & 1.43 & -7.75 & 11.43 & 8,553 \\
& 25 & 0.00 & 1.43 & -7.74 & 11.43 & 8,535 \\
\hline quasi-uniform & 200 & -0.11 & 1.25 & -7.74 & 10.92 & 8,581 \\
& 100 & -0.09 & 1.48 & -8.42 & 11.40 & 8,557 \\
& 50 & -0.02 & 1.43 & -8.22 & 11.43 & 8,525 \\
& 25 & 0.00 & 1.43 & -7.75 & 11.43 & 8,535 \\
\hline Reference & 12.5 & 0.00 & 1.43 & -7.74 & 11.43 & 8,535
\end{tabular}

\subsubsection{Case $\mu=2 \times 10^{-5} \mathrm{~m}^{2} / \mathrm{s}$}

The rising thermal bubble test case is repeated with the viscosity $\mu$ set to $2 \times 10^{-5}$. The first purpose of this test case is simply to demonstrate that the proposed numerical method, implemented with such low viscosity and a $C^{0}$ initial condition, has complete time stability using the same time step and amount of hyperviscosity as is the previous section. Secondly, we are interested in observing how the instability pattern at the leading edge of thermal bubble evolves as the node layout changes. Normally, in numerical testing, to see different evolutions of a solution the initial condition is perturbed. However with RBFs, one has the flexibility of leaving the initial condition intact and perturbing the node layout, which in a turbulent regime will lead to different evolutions of the 
solution. This can be seen in Figure 19. At 200m, there is not much difference between the bubbles. However as can be seen in the $25 \mathrm{~m}$ results, the shear instability layer at the leading edge of the bubble (darkest contours) develops tight eddies whose structure varies significantly depending on the node layout. In both the Cartesian and hexagonal case, the eddy development is completely symmetric about the midpoint of the bubble due to the symmetry in the node layout, while in the quasi-uniform node layout this is not the case (a seemingly more realistic scenario for modeling warm air entrainment in the atmosphere). Furthermore, the scale of the eddies and the degree to which they excite finer scale instabilities varies between the node sets. The Cartesian node layout produces the largest scale eddies as well as the smallest amount of eddies. In contrast, the hexagonal nodes produce a rather strange bubble shape with very fine scale eddy structure. 

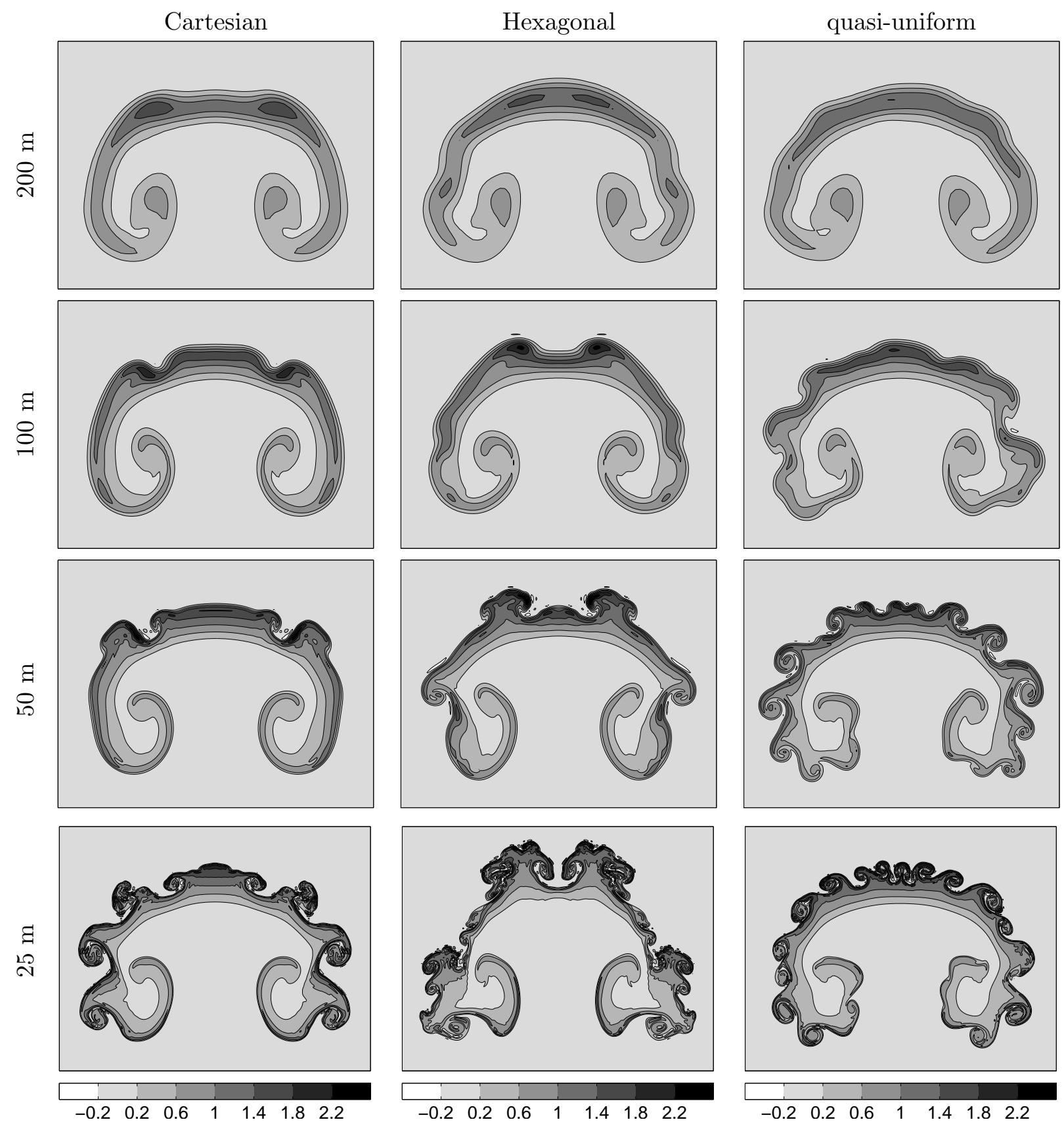

Figure 19: Numerical solutions for the rising thermal bubble with $\mu=2 \times 10^{-5} \mathrm{~m}^{2} / \mathrm{s}$ on the three different types of node distributions at various resolutions. All results are shown at the final simulation time, $t=1100$ s.

\section{Conclusions and summary}

In this paper, a modified RBF-FD method is introduced to construct differentiation weights based on a combined RBF-polynomial basis, using RBF polyharmonic splines $\left(\phi(r)=r^{m}\right)$ with polyno- 
mial functions up to degree $l$ in the given dimension of the problem. The method is applied to three standard test cases in the numerical weather prediction community [3, 43,34], with the latter two based on the Navier-Stokes (NS) equations. In addition, the effect of node layout (Cartesian, hexagonal, or quasi-uniform) on the error as well the qualitative character of the solution is considered. The following observations are made:

1. Under refinement, with the inclusion of polynomials, stagnation (saturation) error is evaded.

2. In the absence of boundary effects, the convergence rate is controlled, not by the order of the PHS RBF, but by the highest degree polynomials used.

3. Increasing the order of the PHS marginally increases the accuracy, as the constant that multiples the convergence rate decreases.

4. For stable configurations that require no tuning of the hyperviscosity (e.g. $\gamma=2^{-6} h^{-2 k}$ for all NS tests), the number of nodes in the stencil, $n$, should be approximately twice the number of polynomial basis functions, $(l+1)(l+2) / 2$ in $2 \mathrm{D}$. Hence on an $n=37$ node stencil, up to fourth-order polynomials ( 15 in $2 \mathrm{D}$ ) are used for the NS equations.

5. For a given polynomial degree, the stencil size has little effect on accuracy.

6. In the absence of boundary effects, for a hyperbolic PDE, neither the character of the solution, the error, nor the convergence rate is sensitive to the node layout.

7. In the presence of boundaries, the solution on Cartesian nodes exhibited significant oscillations (Runge phenomena) near the boundary. This is not the case with hexagonal nodes, which was the most effective node layout in correctly capturing the physics, especially at lower resolutions.

8. In all cases, quasi-uniform nodes showed no detriment to the quality of the solution, error, or convergence and in the majority of the cases performed better than Cartesian layouts. This attribute is important as geometric flexibility of the node layout is crucial when considering local node refinement.

9. Decreasing the viscosity by 6 orders of magnitude, (i.e. increasing in the Reynolds number by the same factor), does not require any change to the time step or amount of hyperviscosity added.

10. In the turbulent regime, the type of node layout heavily impacts the location and structure of eddy development on the leading edge of the thermal bubble as well as the degree of excitation of finer scale instabilities.

Acknowledgements The authors would like to thank Professor Bengt Fornberg, Dr. Victor Bayona, and Mr. Bradley Martin for useful comments and discussions. Dr. Flyer and Mr. Gregory Barnett would like to acknowledge the support of NSF grant DMS-094581. The National Center for Atmospheric Research is sponsored by NSF. 


\section{A Symmetric Stencils}
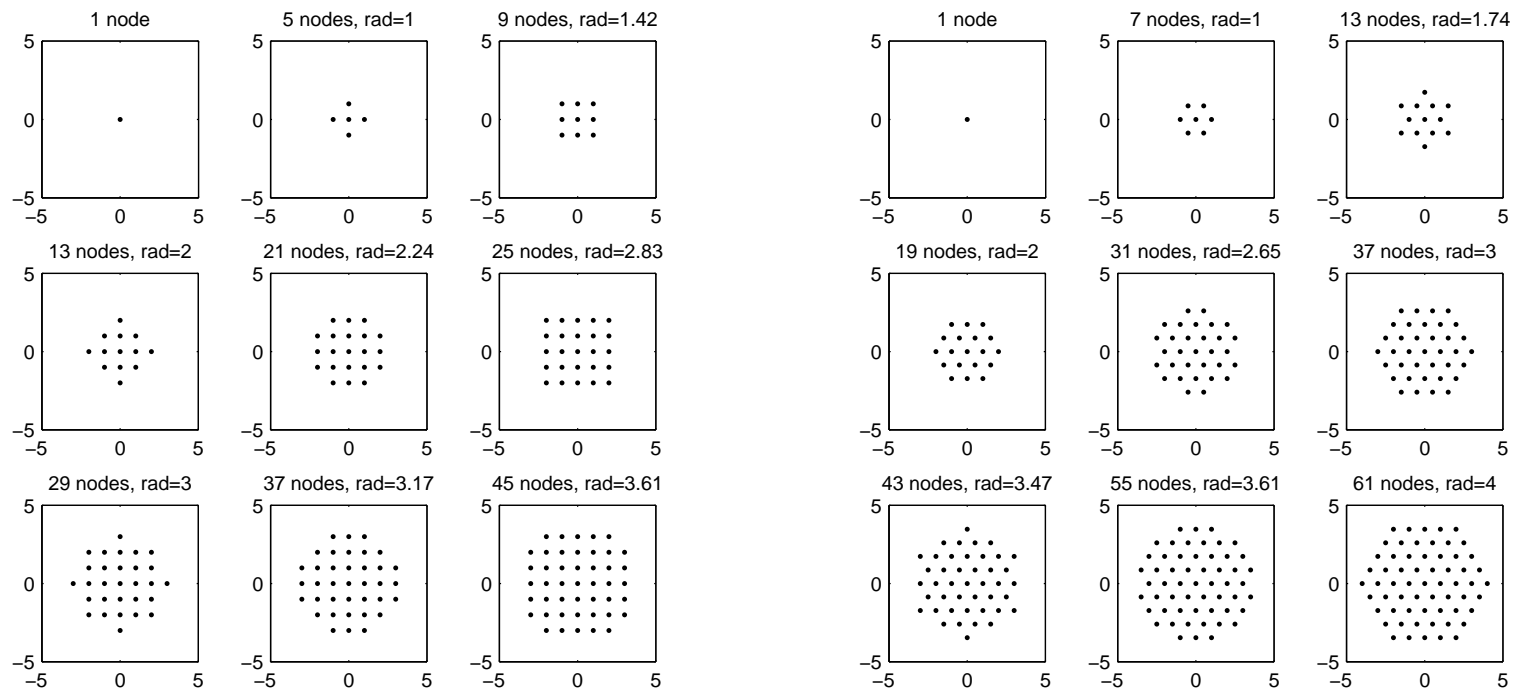

Figure 20: Symmetric stencils for cartesian and hexagonal nodes. Note that 13 and 37 are the only reasonably small stencil-sizes held in common.

\section{B MATLAB code for PHS-polynomial differentiation weights}

Note that in the code all node points $\{x, y\}_{k=1}^{n}$ are translated so the evaluation point, denoted $\mathbf{x}_{\mathbf{c}}$ in Section 3, is the origin $(0,0)$. This greatly simplifies evaluating the right hand side (RHS) in (3). All polynomial terms on the RHS will be zero except a single one that evaluates to either 1 or 2 depending on whether the differentiation weights for a first or second derivative are calculated. Furthermore, since RBFs are radially symmetric, we can instead center them all at the origin and evaluate the RBF part of the RHS at the node locations. This greatly simplifies the algebra, noting a flip in sign for odd derivatives (note the definition of L0 in the code for calculating $\frac{\partial}{\partial x}$ and $\frac{\partial}{\partial y}$ is simply $-\mathrm{x}$ and $-\mathrm{y}$, respectively).

function $w=$ RBF_FD_PHS_pol_weights $(x, y, m, d)$

$\%$ Input parameters

$\% \quad \mathrm{x}, \mathrm{y} \quad$ Column vectors with stencil node locations; approximation to

$\% \quad$ be accurate at $\mathrm{x}(1), \mathrm{y}(1)$

$\% \quad m \quad$ Power of $r$ in $\operatorname{RBF} f i(r)=r^{\wedge} m$, with $m$ odd, $>=3$.

$\%$ d Degree of supplementary polynomials ( $d=-1$ no polynomials)

$\%$

$\%$ Output parameter

$\%$ w Matrix with three columns, containing weights for $d / d x, d / d y$, $\% \quad$ and the Laplacian $\mathrm{d} 2 / \mathrm{dx} 2+\mathrm{d} 2 / \mathrm{dy} 2$, respectively. 


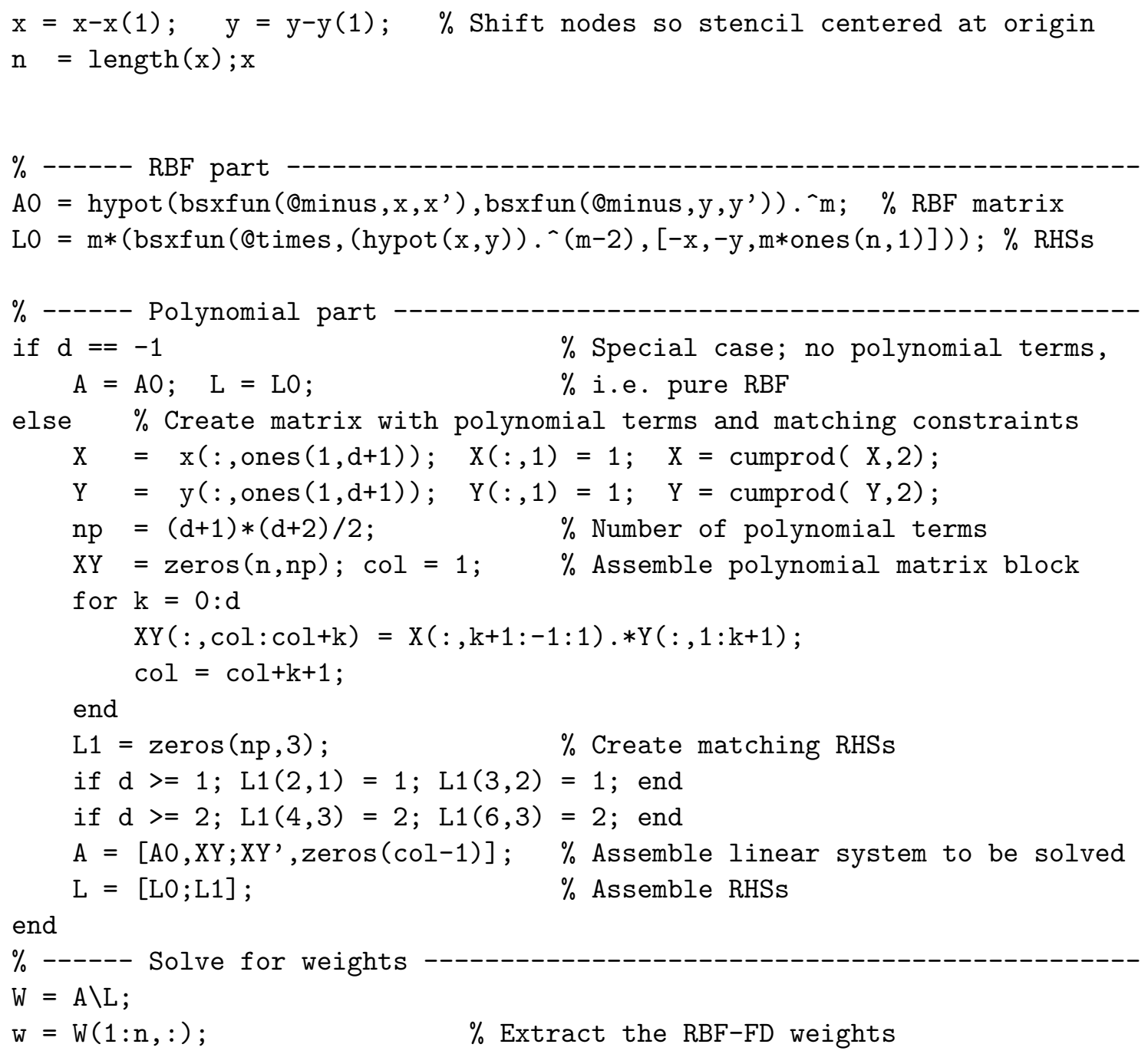

\section{References}

[1] V. Bayona, N. Flyer, and B. Fornberg, On the role of polynomials in RBF-FD approximations: Ii. numerical solution of elliptic PDEs, (2016), to be submitted.

[2] V. Bayona, M. Moscoso, and M. Kindelan, Optimal constant shape parameter for multiquadric based RBF-FD method, J. Comput. Phys. 230 (2011), 7384-7399.

[3] P. N. Blossey and D. R. Durran, Selective monotonicity preservation in scalar advection, J. Comput. Phys. 227(10) (2008), 5160-5183.

[4] E. Bollig, N. Flyer, and G. Erlebacher, Solution to PDEs using radial basis function finitedifferences (RBF-FD) on multiple GPUs, J. Comput. Phys. 231 (2012), 7133-7151.

[5] A. H.-D. Cheng, Multiquadric and its shape parameter - A numerical investigation of error estimate, condition number, and round-off error by arbitrary precision computation, Eng. Anal. Bound. Elem. 36 (2012), 220-239. 
[6] O. Davydov and D. T. Oanh, On the optimal shape parameter for Gaussian radial basis function finite difference approximation of the Poisson equation, Comp. Math. with Appl. 62 (2011), 2143-2161.

[7] T. A. Driscoll and B. Fornberg, Interpolation in the limit of increasingly flat radial basis functions, Comput. Math. Appl. 43 (2002), 413-422.

[8] J. Duchon, Splines mimimizing rotation-invariant semi-norms in Sobolev space, Constructive Theory of Functions of Several Variables, Springer Lecture Notes in Math 21 (1977), 85-100.

[9] J.C. Fabero, A. Bautista, and L. Casasús, An explicit finite differences scheme over hexagonal tessellation, Appl. Math Lett. 14 (2001), 593-598.

[10] G. E. Fasshauer, Meshfree Approximation Methods with MATLAB, Interdisciplinary Mathematical Sciences - Vol. 6, World Scientific Publishers, Singapore, 2007.

[11] G. E. Fasshauer and J. G. Zhang, On choosing "optimal" shape parameters for RBF approximation, Num. Alg. 45 (2007), 345-368.

[12] N. Flyer, B. Fornberg, V. Bayona, and G. A. Barnett, On the role of polynomials in RBF-FD approximations: Interpolation and accuracy, JCP submitted (2016).

[13] N. Flyer, E. Lehto, S. Blaise, G. B. Wright, and A. St-Cyr, A guide to RBF-generated finite differences for nonlinear transport: Shallow water simulations on a sphere, J. Comput. Phys 231 (2012), 4078-4095.

[14] T. A. Foley, Near optimal parameter selection for multiquadric interpolation, J. Appl. Sci. Comput. 1 (1994), 54-69.

[15] B. Fornberg, T. A. Driscoll, G. Wright, and R. Charles, Observations on the behavior of radial basis functions near boundaries, Comput. Math. Appl. 43 (2002), 473-490.

[16] B. Fornberg and N. Flyer, Fast generation of 2-D node distributions for mesh-free PDE discretizations, Comp. Math. Appl. 69 (2015), 531-544.

[17] _ A Primer on Radial Basis Functions with Applications to the Geosciences, SIAM, Philadelphia, 2015.

[18] B. Fornberg and E. Lehto, Stabilization of RBF-generated finite difference methods for convective PDEs, J. Comput. Phys. 230 (2011), 2270-2285.

[19] B. Fornberg, E. Lehto, and C. Powell, Stable calculation of Gaussian-based RBF-FD stencils, Comp. Math. Applic. 65 (2013), 627-637.

[20] B. Fornberg, G. Wright, and E. Larsson, Some observations regarding interpolants in the limit of flat radial basis functions, Comput. Math. Appl. 47 (2004), 37-55.

[21] F. X. Giraldo and M. Restelli, A study of spectral element and discontinuous Galerkin methods for the navier-stokes equations in nonhydrostatic mesoscale atmospheric modeling: Equation sets and test cases, J. Comput. Phys. 227 (2008), 3849-3877.

[22] W.W. Grabowski and T.L. Clark, Cloud-environment interface instability: Rising thermal calculations in two spatial dimensions, Mon. Wea. Rev. 48(4) (1991), 527-546. 
[23] B. Hamilton and S. Bilbao, Hexagonal vs. rectilinear grids for explicit finite difference schemes for the two-dimensional wave equation, Proceedings of Meetings on Acoustics (POMA) (Montreal, Canada), Acoustical Society of America, 2013, pp. POMA 19, 015120.

[24] C.-S. Huang, C.-F. Leeb, and A. H.-D. Cheng, Error estimate, optimal shape factor, and high precision computation of multiquadric collocation method, Eng. Anal. Bound. Elem. 31 (2007), 614-623.

[25] A. Iske, On the approximation order and numerical stability of local Lagrange interpolation by polyharmonic splines, Modern Developments in Multivariate Approximation (W. Haussmann, K. Jetter, M. Reimer, and J. Stöckler, eds.), International Series of Numerical Mathematics, vol. 145, Birkhäuser Verlag, Basel, 2003, pp. 153-165.

[26] _ Multiresolution Methods in Scattered Data Modelling, Lecture Notes in Computational Science and Engineering, vol. 37, Springer-Verlag, Heidelberg, 2004.

[27] E. Larsson, E. Lehto, A. Heryudono, and B. Fornberg, Stable computation of differentiation matrices and scattered node stencils based on Gaussian radial basis functions, SIAM J. Sci. Comput. 35 (2013), A2096-A2119.

[28] D. Lee, H.C. Tien, C.P. Luo, and H.-N. Luk, Hexagonal grid methods with applications to partial differential equations, Int. J. Comp. Math. 91 (2014), 1986-2009.

[29] R. J. LeVeque, High-resolution conservative algorithms for advection in incompressible flow, SIAM J. Numer. Anal 33 (1996), 627-665.

[30] B. Matérn, Stochastic models and their application to some problems in forest surveys and other sampling investigations, Ph.D. thesis, University of Stockholm, Stockholm, Sweden, June 1960.

[31] M. R. Norman, R. D. Nair, and F. H. M. Semazzi, A low communication and large time step explicit finite-volume solver for non-hydrostatic atmospheric dynamics, J. Comput. Phys. 230 (2011), 1567-1584.

[32] K.V. Ooyama, A dynamic and thermodynamic foundation for modeling the moist atmosphere with parameterized microphysics, J. Atm. Sci 58 (2001), 2072-2102.

[33] M. J. D. Powell, The theory of radial basis function approximation in 1990, Advances in Numerical Analysis, Vol. II: Wavelets, Subdivision Algorithms and Radial Functions (W. Light, ed.), Oxford University Press, Oxford, UK, 1992, pp. 105-210.

[34] A. Robert, Bubble convection experiments with a semi-implicit formulation of the euler equations, J. Atmosph. Sci. 50 (1993), 1865-1873.

[35] R. Schaback, Error estimates and condition numbers for radial basis function interpolants, Adv. Comput. Math. 3 (1995), 251-264.

[36] _ Multivariate interpolation by polynomials and radial basis functions, Constr. Approx. 21 (2005), 293-317.

[37] M. Scheuerer, An alternative procedure for selecting a good value for the parameter $c$ in $R B F$ interpolation, Adv. Comput. Math. 34 (2011), 105-126.

[38] Y. Y. Shan, C. Shu, and Z. L. Lu, Application of local MQ-DQ method to solve 3D incompressible viscous flows with curved boundary, Comp. Mod. Eng. \& Sci. 25 (2008), 99-113. 
[39] Y. Y. Shan, C. Shu, and N. Qin, Multiquadric finite difference (MQ-FD) method and its application, Adv. Appl. Math. Mech. 1 (2009), 615-638.

[40] W. C. Skamarock, Positive-definite and monotonic limiters for unrestricted-time-step transport schemes, Mon. Wea. Rev 24 (2006), 22412250.

[41] W. C. Skamarock and J. B. Klemp, A time-split nonhydrostatic atmospheric model for weather research and forecasting applications, J. Inst. Math. Appl. 227 (2008), 3465-3485.

[42] D. Stevens, H. Power, M. Lees, and H. Morvan, The use of PDE centers in the local RBF Hermitean method for $3 D$ convective-diffusion problems, J. Comput. Phys. 228 (2009), 46064624 .

[43] J.M. Straka, R.B. Wilhelmson, L.J. Wicker, J.R. Anderson, and K.K. Droegemeier, Numerical solutions of a nonlinear density current: a benchmark solution and comparisons, Int. J. Num. Meth. Fluids 17 (1993), 1-22.

[44] A. E. Tarwater, Parameter study of Hardy's multiquadric method for scattered data interpolation, Technical Report UCRL-54670, Lawrence Livermore National Laboratory, 1985.

[45] H. Wendland, Piecewise polynomial, positive definite and compactly supported radial functions of minimal degree, Adv. Comput. Math. 4 (1995), 389-396.

[46] _ Scattered Data Approximation, Cambridge Monographs on Applied and Computational Mathematics, vol. 17, Cambridge University Press, Cambridge, 2005.

[47] L. J. Wicker and W.C. Skamarock, Time-splitting methods for elastic models using forward time schemes, Mon. Wea. Rew. 130 (2002), 2088-2097. 